\title{
Fire and Life Safety Evaluation
}

197 Bonderson Engineering Project Center

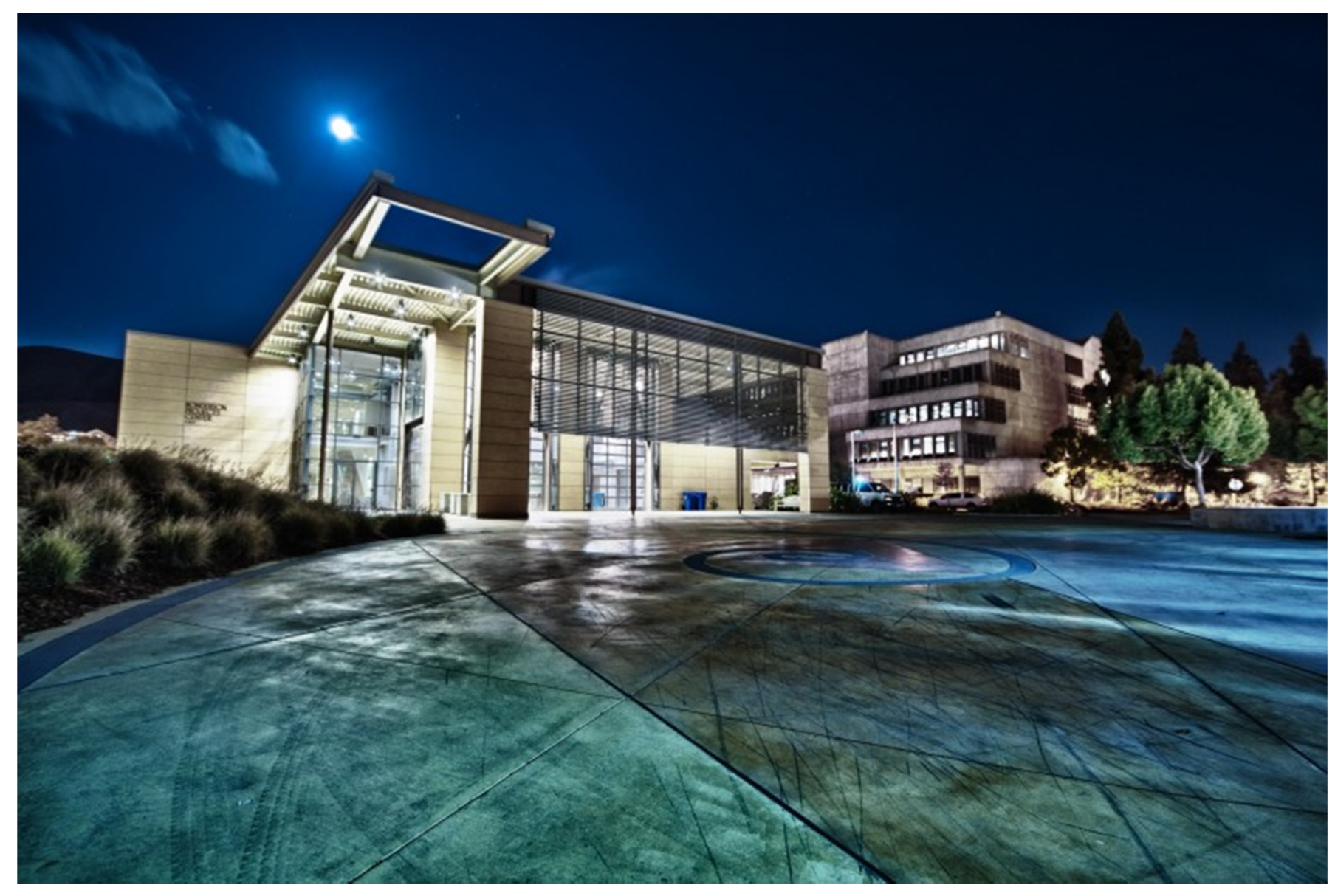

Gary Dominguez 


\section{Contents}

- Applicable Codes

- Building Location and Description

- Structural Fire Protection

- Egress Analysis

- Fire Suppression System

- Fire Detection and Alarm

- Performance Based Analysis 


\section{Applicable Codes}

- California Building Code (CBC), 2013 Edition

- California Fire Code (CFC), 2013 Edition

- NFPA 13 Standard for the Installation of Sprinkler Systems, 2013 Edition

- NFPA 72 National Fire Alarm and Signaling Code, 2013 Edition

- SFPE Handbook of Fire Protection Engineering, Fourth Edition 


\section{Building Location}

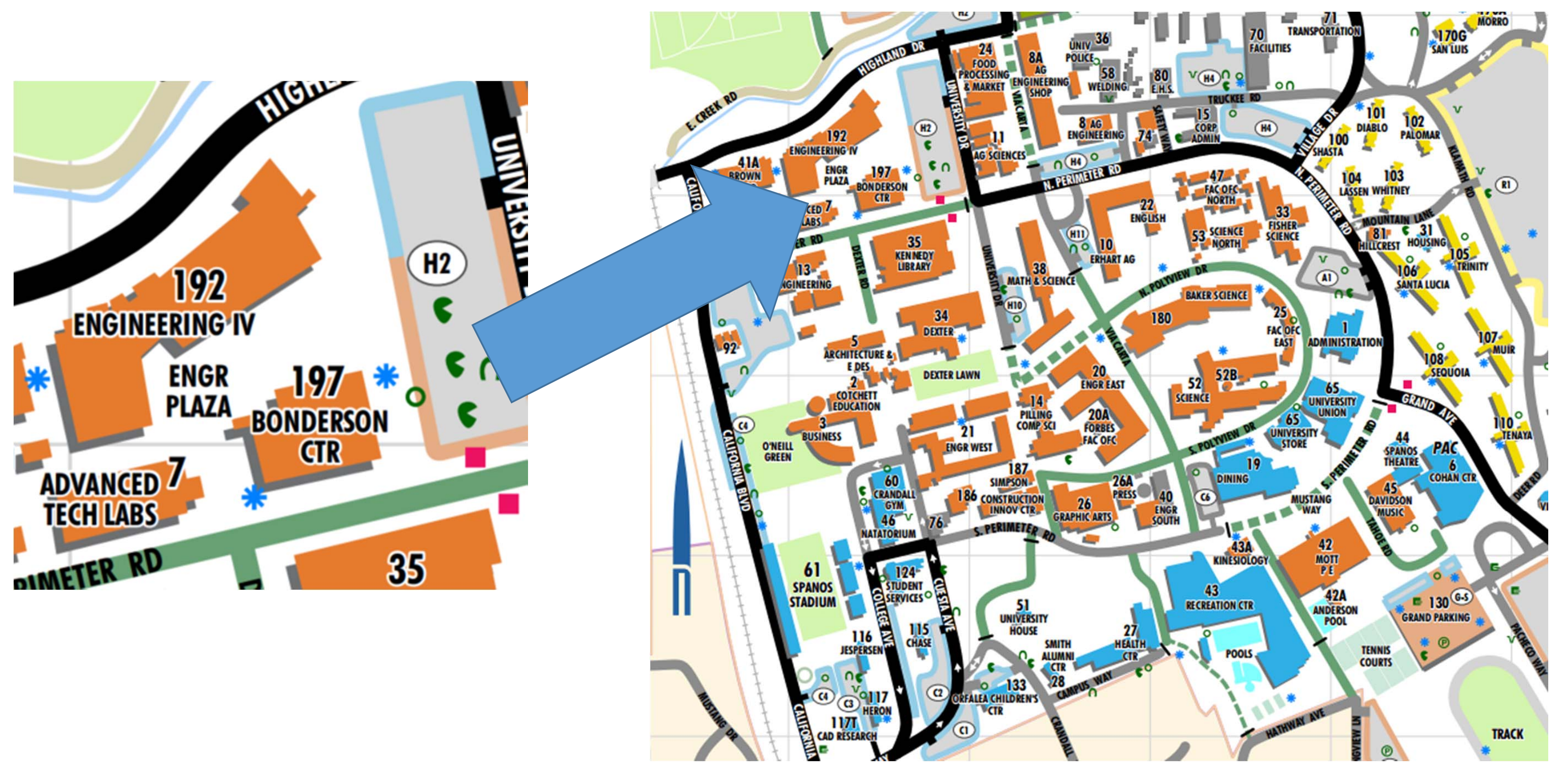




\section{Building Description}

- Building Area: $1^{\text {st }}$ Floor- $12,240 \mathrm{ft}^{2}$

$$
2^{\text {nd }} \text { Floor- 6,335 } \mathrm{ft}^{2}
$$

Total- $18,575 \mathrm{ft}^{2}$

- Two-Story Building

- Concrete Slab and Steel Frame Construction

- Construction Type: II-B

- Fully Sprinklered

- Equipped with Fire Detection and Alarm Notification Devices

- Type B Occupancy

- Atrium at Entry 


\section{Structural Requirements}

\begin{tabular}{|l|c|}
\hline \multicolumn{2}{|c|}{ CBC Table 601 } \\
\hline \multicolumn{1}{|c|}{ Building Element } & $\begin{array}{c}\text { Fire-Resistance Rating } \\
\text { Requirement (Hours) }\end{array}$ \\
\hline Primary Structural frame & 0 \\
\hline Bearing walls (exterior) & 0 \\
\hline Bearing walls (interior) & 0 \\
\hline $\begin{array}{l}\text { Nonbearing walls and partitions } \\
\text { (interior) }\end{array}$ & 0 \\
\hline $\begin{array}{l}\text { Floor construction and associated } \\
\text { secondary members }\end{array}$ & 0 \\
\hline $\begin{array}{l}\text { Roof construction and associated } \\
\text { secondary members }\end{array}$ & 0 \\
\hline
\end{tabular}

\section{CBC Table 602}

\begin{tabular}{|l|c|c|}
\hline \multirow{2}{*}{ Fire Separation Distance $-X$ (feet) } & \multicolumn{2}{|c|}{$\begin{array}{r}\text { Fire-Resistance Rating Requirement } \\
\text { for Exterior Walls (Hours) }\end{array}$} \\
\cline { 2 - 3 } & Group F-1 & Group A, B, S-2 \\
\hline$X<5$ & 2 & 1 \\
\hline $5 \leq X<10$ & 1 & 1 \\
\hline $10 \leq X<30$ & 0 & 0 \\
\hline$X \geq 30$ & 0 & 0 \\
\hline
\end{tabular}

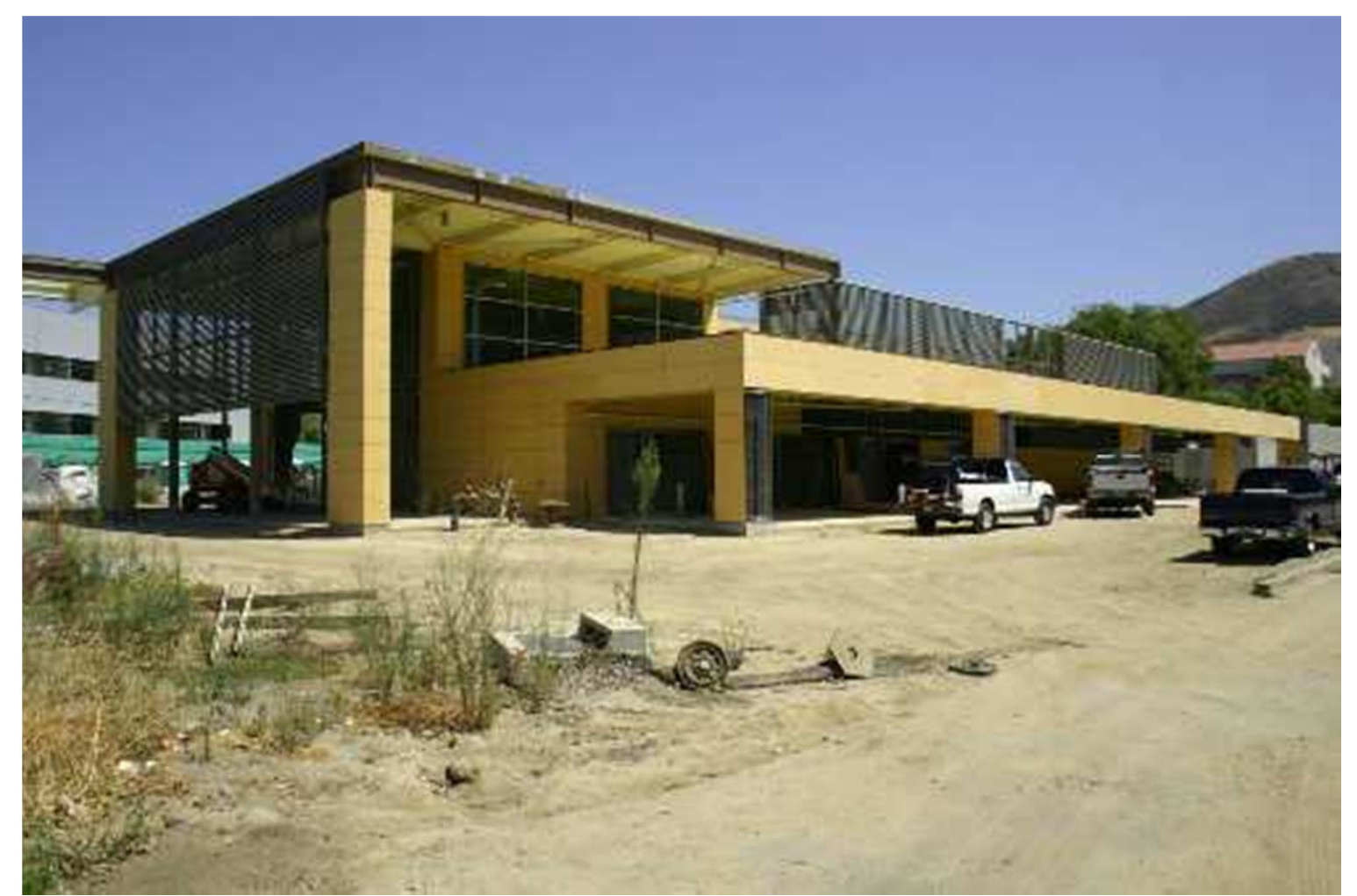




\section{Interior Finishes}

CBC Table 803.9

\begin{tabular}{|c|c|c|c|}
\hline Group & $\begin{array}{c}\text { Interior exit stairways, interior exit } \\
\text { ramps and exit passageways }\end{array}$ & $\begin{array}{c}\text { Corridors and enclosure for exit acces } \\
\text { stairways and exit access ramps }\end{array}$ & Room and enclosed spaces \\
\hline A-3 & B & B & C \\
\hline B & B & C & C \\
\hline F & C & C & C \\
\hline S & C & C & C \\
\hline
\end{tabular}

Class A: = Flame spread index 0-25; smokedeveloped index 0-450.

Class B: $=$ Flame spread index 26-75; smokedeveloped index 0-450.

Class C: = Flame spread index 76-200; smokedeveloped index 0-450.

- Interior finish of walls and ceilings of atrium must not be less than Class B 


\section{Occupancy Classification}

\begin{tabular}{|c|c|}
\hline \multicolumn{2}{|c|}{ CBC Table 1021.1 } \\
\hline Occupant Load per Story & Minimum Number of Exits \\
\hline $1-500$ & 2 \\
\hline
\end{tabular}

CBC Section 1005

\begin{tabular}{|l|c|}
\hline \multicolumn{1}{|c|}{ Component } & $\begin{array}{c}\text { Egress Capacity Factor } \\
\text { (inch per person) }\end{array}$ \\
\hline Stairways & 0.3 \\
\hline Other egress components & 0.2 \\
\hline
\end{tabular}

\begin{tabular}{|l|c|}
\hline \multicolumn{2}{|c|}{ CBC Table 1004.1.2 } \\
\hline \multicolumn{1}{|c|}{ Classification } & $\begin{array}{c}\text { Occupant Load Factor } \\
\left(\mathrm{ft}^{2} \text { per occupant }\right)\end{array}$ \\
\hline $\begin{array}{l}\text { Assembly without fixed seats } \\
\text { (Unconcentrated) }\end{array}$ & 15 \\
\hline Classroom Area & 20 \\
\hline Shop and other vocational rooms & 50 \\
\hline $\begin{array}{l}\text { Offices } \\
\text { Accessory storage areas, mechanical } \\
\text { equipment room }\end{array}$ & 100 \\
\hline
\end{tabular}




\section{$1^{\text {st }}$ Floor}

\begin{tabular}{|l|l|}
\hline \multicolumn{1}{|c|}{ Classification } & Color \\
\hline Classroom Area & \\
\hline $\begin{array}{l}\text { Shop and other vocational } \\
\text { rooms }\end{array}$ & \\
\hline Offices & \\
\hline Assembly & \\
\hline $\begin{array}{l}\text { Accessory storage areas, } \\
\text { mechanical equipment room }\end{array}$ & \\
\hline
\end{tabular}

\begin{tabular}{|l|c|}
\hline Area & $12,240 \mathrm{ft}^{2}$ \\
\hline Occupant Load & 240 \\
\hline Egress Capacity & 1,620 \\
\hline Required Number of Exits & 2 \\
\hline Number of Exits & 6 \\
\hline
\end{tabular}

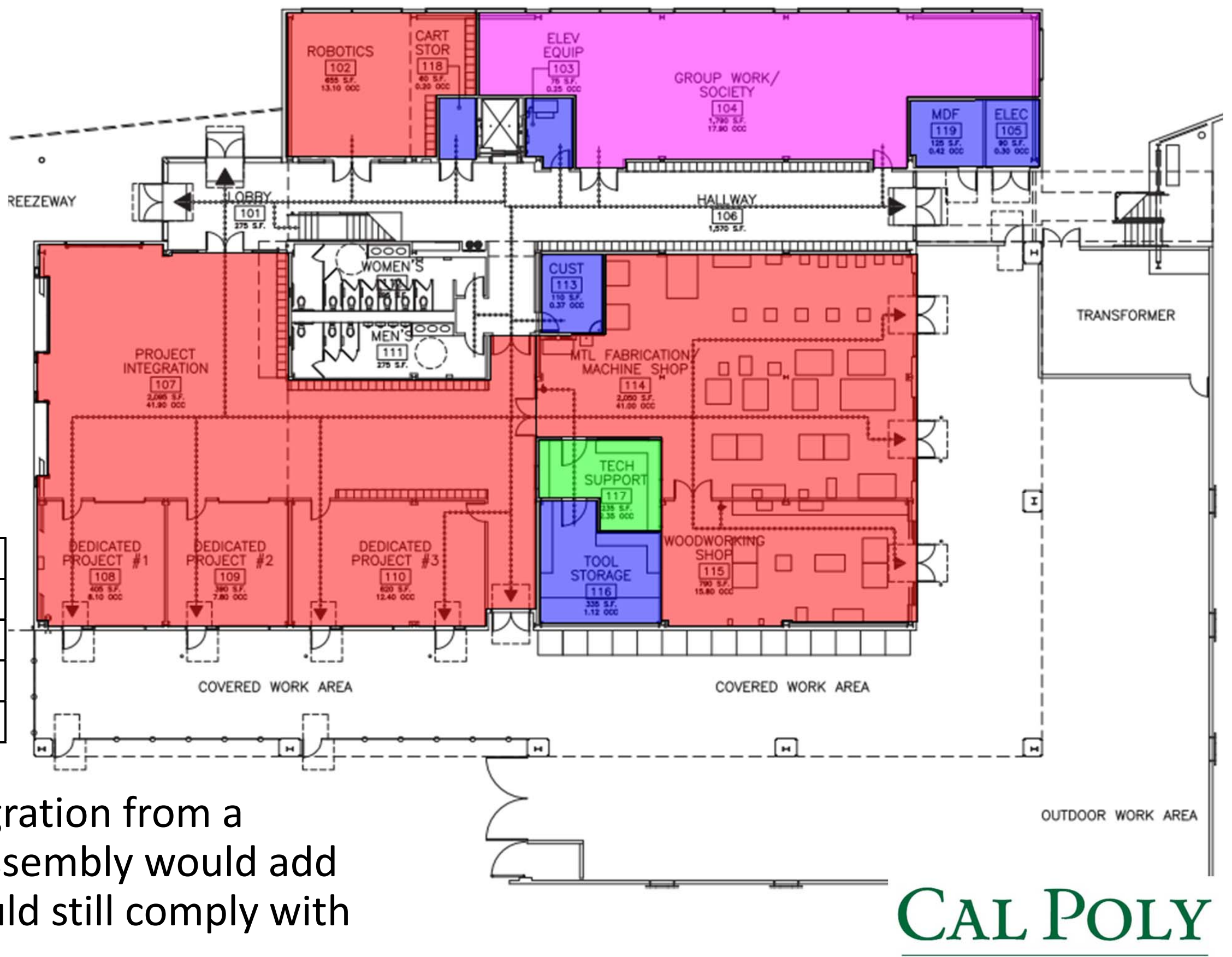

- Changing Project Integration from a Vocational Room to Assembly would add 98 occupants, but would still comply with code 


\section{Project Integration Room}
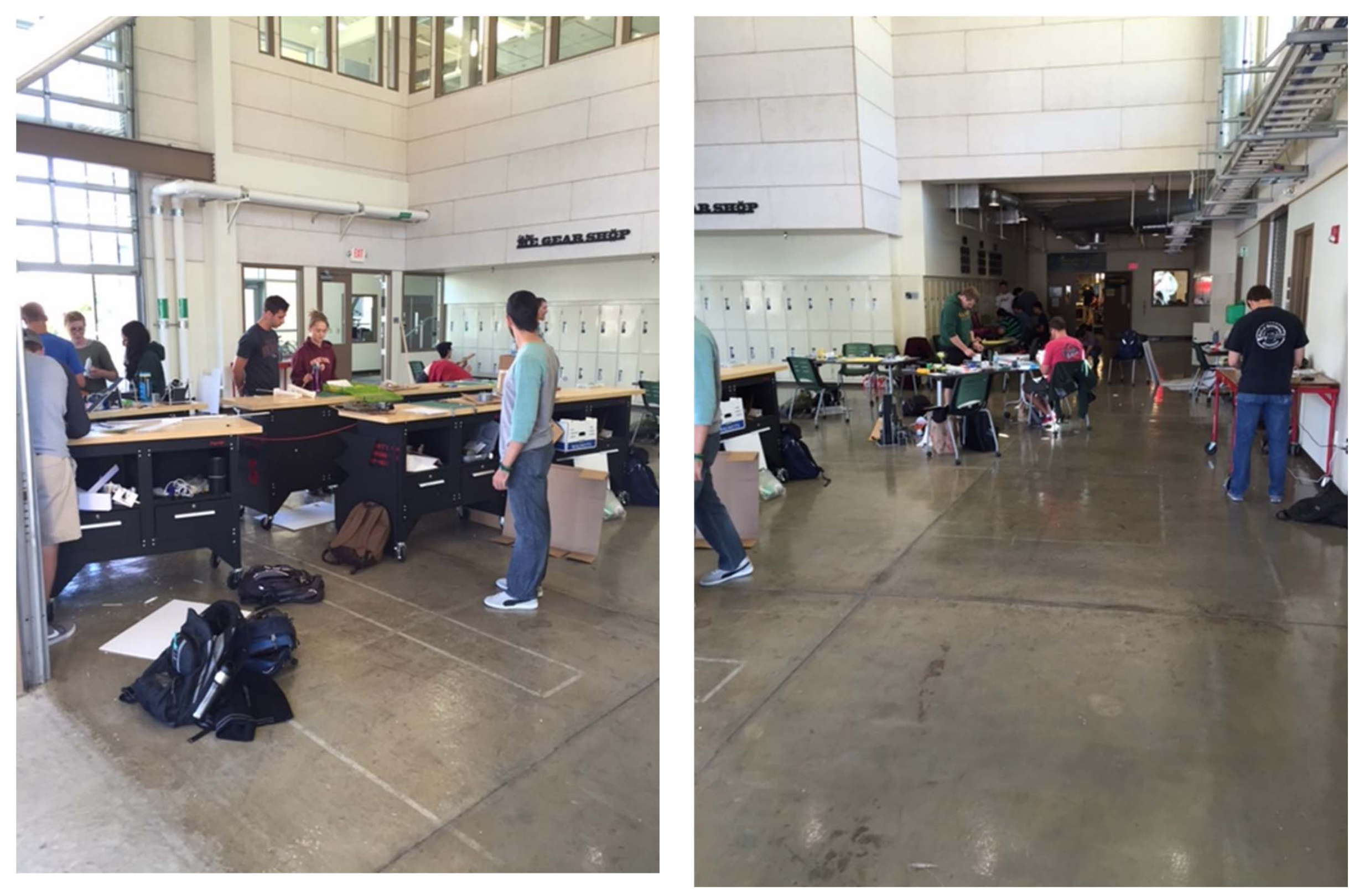

Cal Poly 


\section{Door Swing}

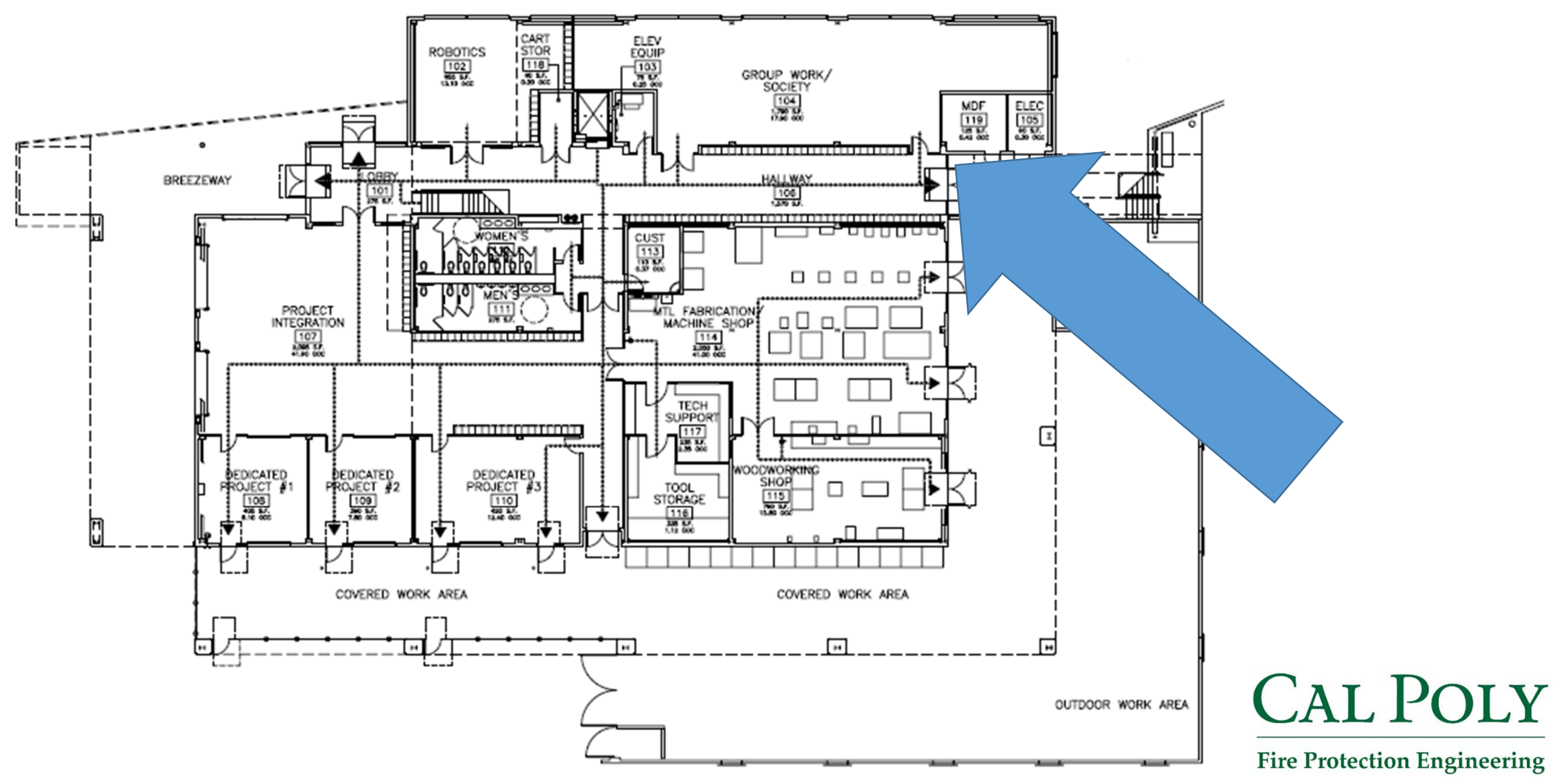


$2^{\text {nd }}$ Floor

\begin{tabular}{|l|l|}
\hline \multicolumn{1}{|c|}{ Classification } & Color \\
\hline Classroom Area & \\
\hline $\begin{array}{l}\text { Shop and other vocational } \\
\text { rooms }\end{array}$ & \\
\hline Offices & \\
\hline Assembly & \\
\hline $\begin{array}{l}\text { Accessory storage areas, } \\
\text { mechanical equipment room }\end{array}$ & \\
\hline
\end{tabular}

\begin{tabular}{|l|c|}
\hline Area & $6,335 \mathrm{ft}^{2}$ \\
\hline Occupant Load & 171 \\
\hline Egress Capacity & 320 \\
\hline Required Number of Exits & 2 \\
\hline Number of Exits & 2 \\
\hline
\end{tabular}

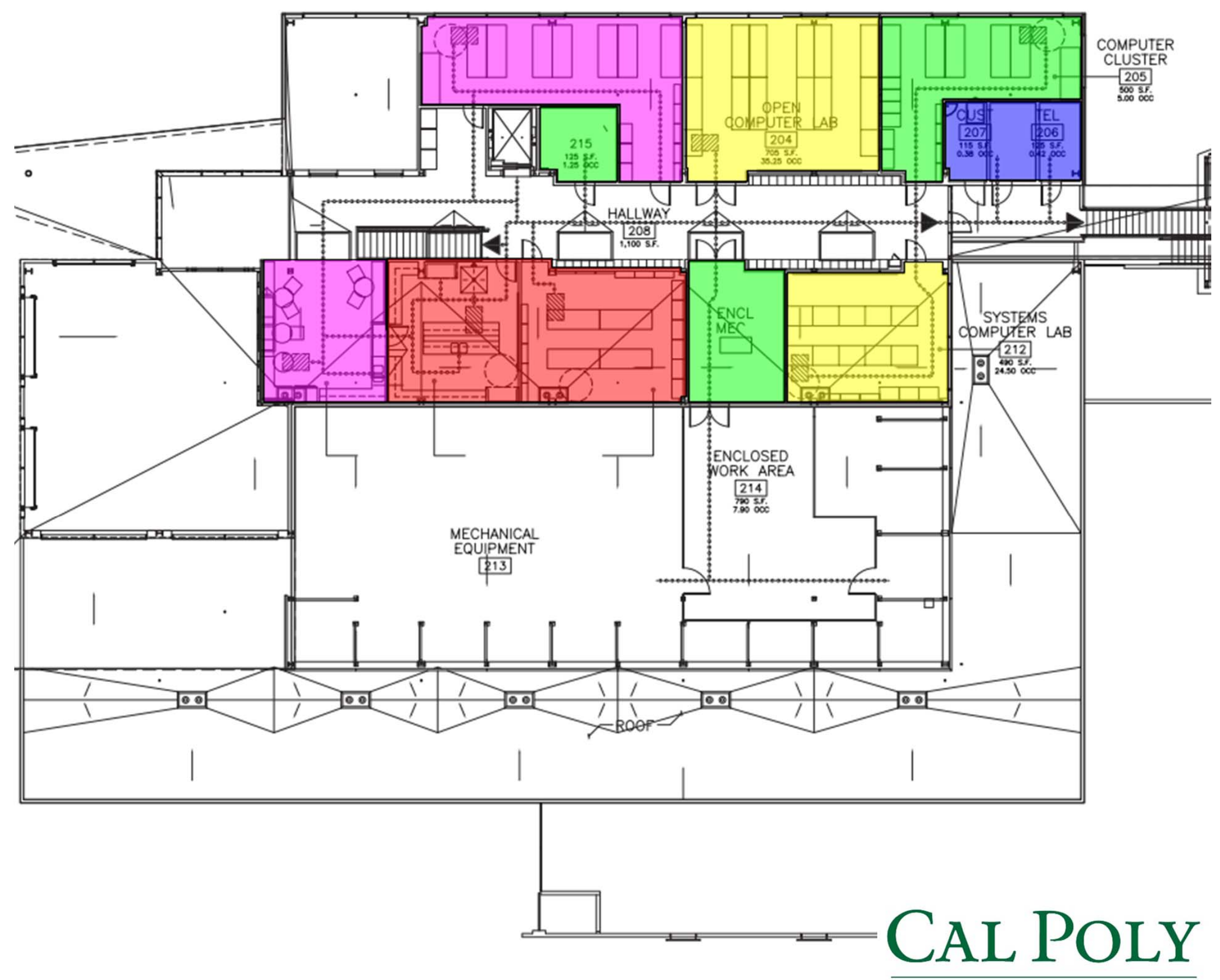

Fire Protection Engineering 


\section{Exit Locations}

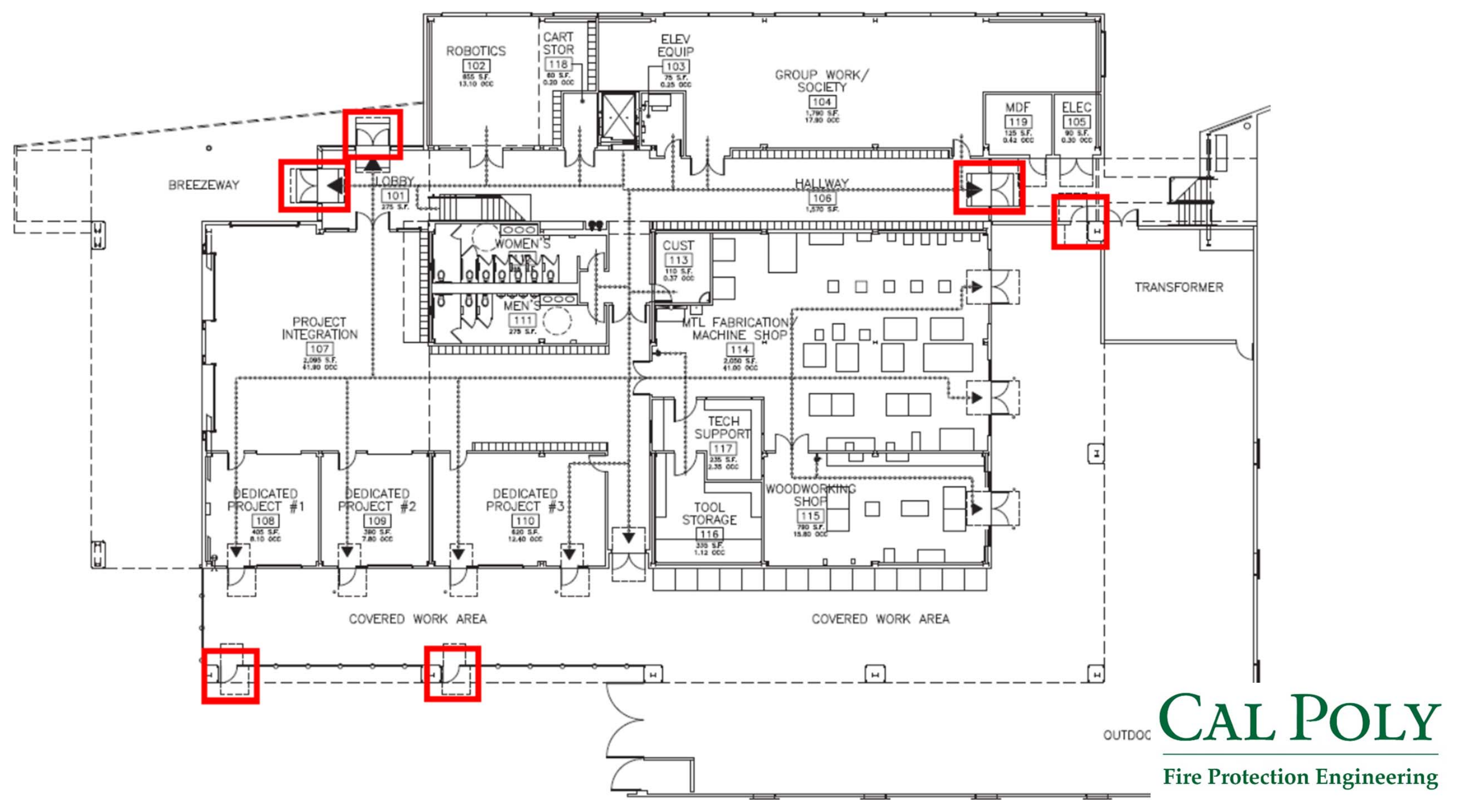




\section{Exit Locations}

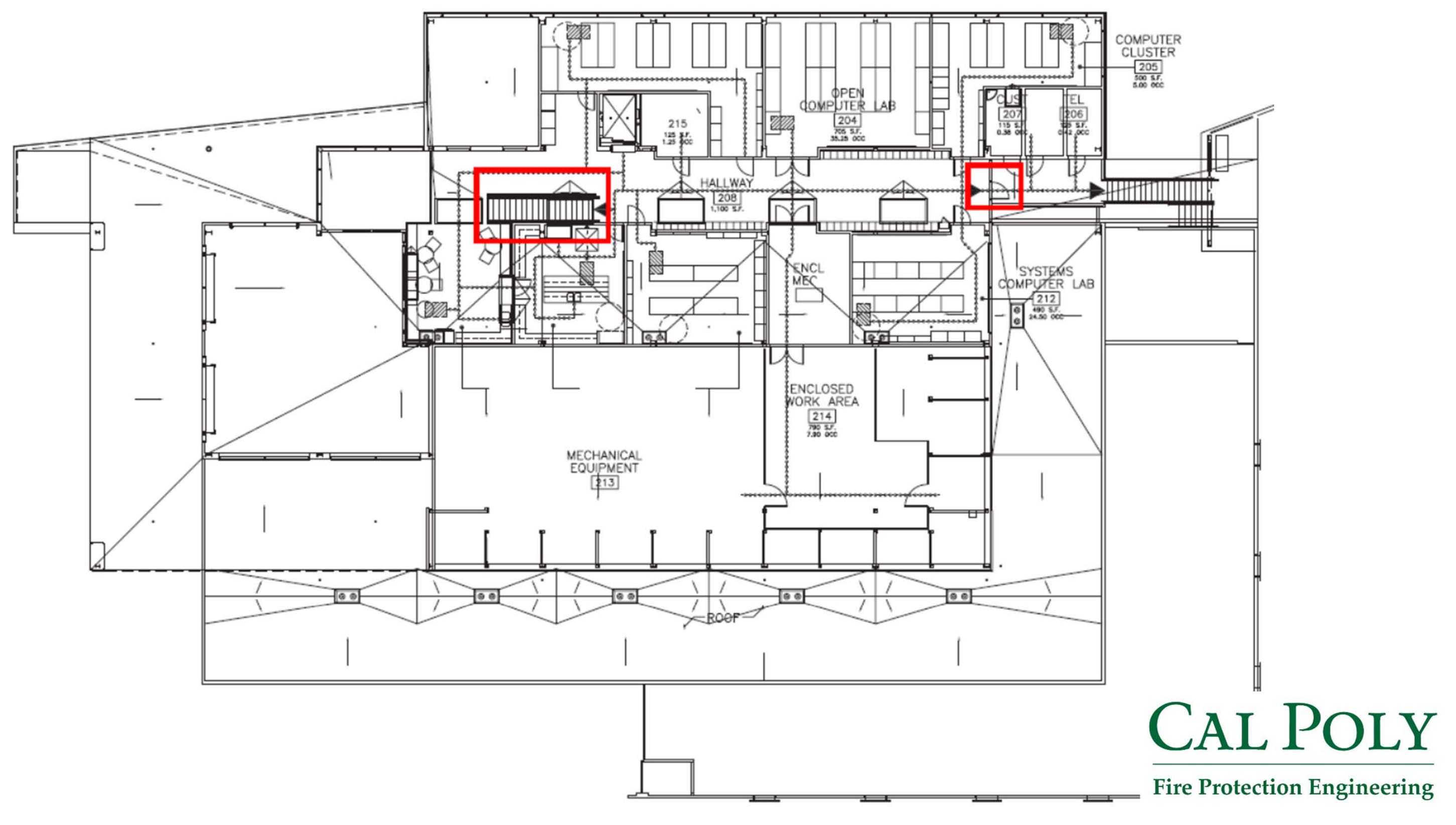




\section{Interior Stairway}

- Exception to required enclosed exit access stairways between stories (CBC 1009.3 Exception 5).

- Portion of exit access travel distance must be less than 200 feet (CBC 404.9) 


\section{Egress Requirements}

Table 1016.2

\begin{tabular}{|c|c|}
\hline Occupancy & Exit Access Travel Distance (feet) \\
\hline A, F-1 & 250 \\
\hline B & 300 \\
\hline S-2 & 400 \\
\hline
\end{tabular}

Table 1014.3

\begin{tabular}{|c|c|}
\hline Occupancy & Common Path of Egress Travel (feet) \\
\hline B & 100 \\
\hline A, F, S & 75 \\
\hline
\end{tabular}

- Building complies with exit access travel distance and common path of egress travel.

- No dead ends in corridors more than 20 feet in length. 


\section{Required Occupancy Separation}

\begin{tabular}{|c|c|c|c|}
\hline \multicolumn{5}{|c|}{ CBC Table 508.4 } \\
\hline Occupancy & A & S-2 & B, F-1 \\
\hline A & 0 & 0 & 1 \\
\hline S-2 & & 0 & 1 \\
\hline B, F-1 & & & 0 \\
\hline
\end{tabular}

\begin{tabular}{|c|c|}
\hline \multicolumn{2}{|c|}{ CBC Table 1018.1 } \\
\hline Occupancy & Required Fire-Resistance Rating \\
\hline A , B , S, F & 0 \\
\hline
\end{tabular}

- Does not comply with separated occupancies.

- No fire-resistance rated corridor required per Table 1018.1.

- 1 Hour Fire Barrier must separate atrium spaces from adjacent spaces (CBC 404.6)

- A fire barrier is not required between the atrium and the adjoining spaces provided that such spaces are accounted for in the design of the smoke control system (CBC 404.6 Exception 3). 


\section{$1^{\text {st }}$ Floor Separation}

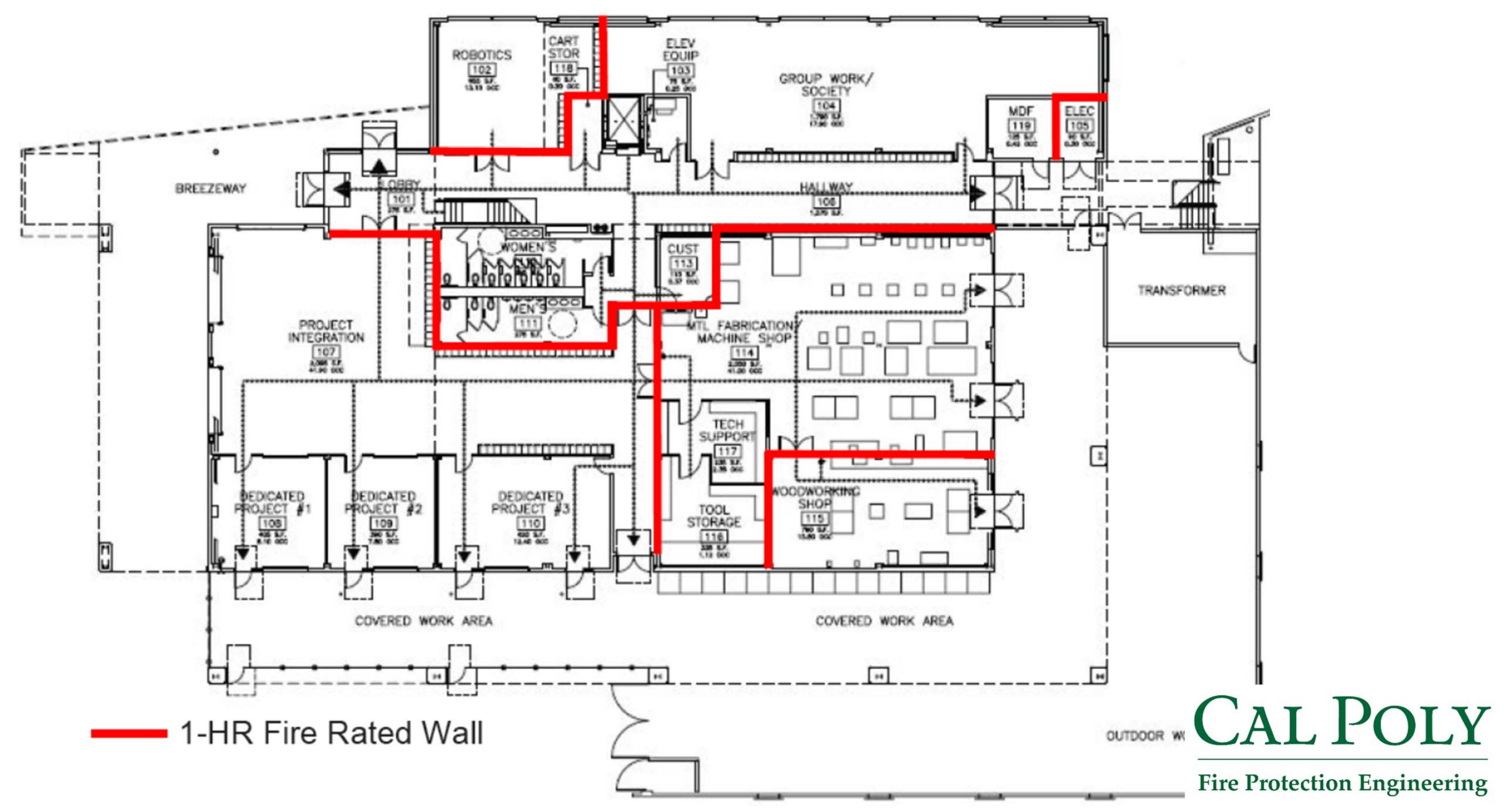




\section{$2^{\text {nd }}$ Floor Separation}

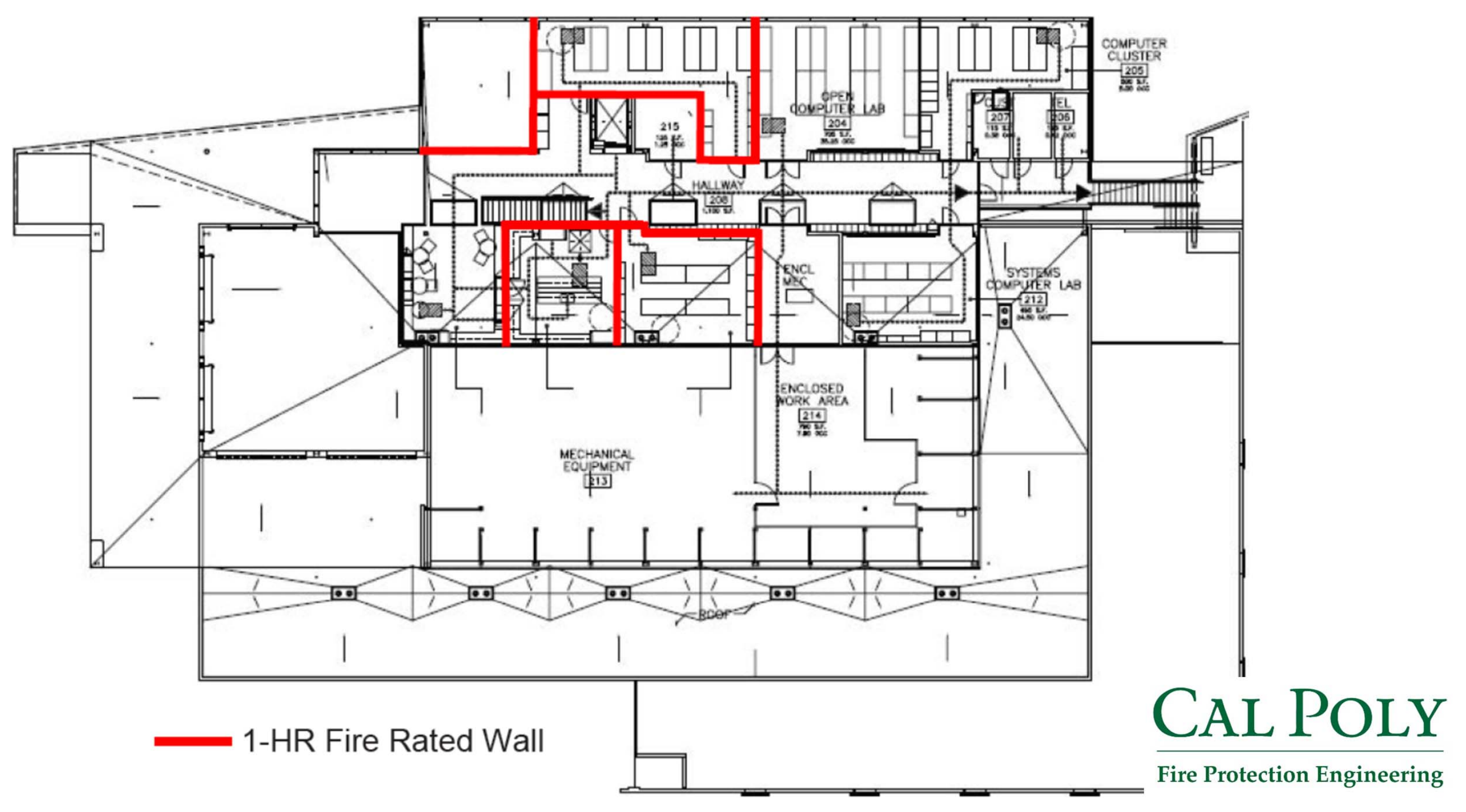




\section{Allowable Height and Area}

\begin{tabular}{|c|c|c|c|c|c|c|c|c|c|c|}
\hline \multirow{6}{*}{ Group } & & \multicolumn{9}{|c|}{ Type of Construction } \\
\hline & & \multicolumn{2}{|c|}{ Type I } & \multicolumn{2}{|c|}{ Type II } & \multicolumn{2}{|c|}{ Type III } & Type IV & \multicolumn{2}{|c|}{ Type V } \\
\hline & & A & B & A & B & A & B & HT & A & B \\
\hline & Height (feet) & UL & 160 & 65 & 55 & 65 & 55 & 65 & 50 & 40 \\
\hline & \multicolumn{10}{|c|}{ Stories (S) } \\
\hline & \multicolumn{10}{|c|}{ Area $(A)$} \\
\hline & $\mathrm{s}$ & UL & 11 & 3 & 2 & 3 & 2 & 3 & 2 & 1 \\
\hline Group A-3 & A & UL & UL & 15,500 & 9,500 & 14,000 & 9,500 & 15,000 & 11,500 & 6,000 \\
\hline & $\mathrm{S}$ & UL & 11 & 5 & 3 & 5 & 3 & 5 & 3 & 2 \\
\hline Group B & $A$ & UL & UL & 37,500 & 23,000 & 28,500 & 19,000 & 36,000 & 18,000 & 9,000 \\
\hline & $S$ & $U L$ & 11 & 4 & 2 & 3 & 2 & 4 & 2 & 1 \\
\hline Group F-1 & $A$ & UL & UL & 25,000 & 15,500 & 19,000 & 12,000 & 33,500 & 14,000 & 8,500 \\
\hline & $\mathrm{S}$ & UL & 11 & 5 & 3 & 4 & 3 & 5 & 4 & 2 \\
\hline Group S-2 & A & UL & 79,000 & 39,000 & 26,000 & 39,000 & 26,000 & 38,500 & 21,000 & 13,500 \\
\hline
\end{tabular}

Building Height: 2 Stories

$1^{\text {st }}$ Floor Area: $12,240 \mathrm{ft}^{2}$ 


\section{Frontage Area Increase}

$I_{f}=[F / P-0.25] W / 30$

(Equation 5-2)

where:

$I_{f}=$ Area increase due to frontage.

$F=$ Building perimeter that fronts on a public way or open space having 20 feet $(6096 \mathrm{~mm})$ open minimum width (feet).

$P=$ Perimeter of entire building (feet).

$W=$ Width of public way or open space (feet) in accordance with Section 506.2.1.

$$
\begin{aligned}
& I_{f}=\left[\frac{F}{P}-0.25\right] \frac{W}{30} \\
& I_{f}=[1.0-0.25] \frac{30}{30} \\
& I_{f}=75 \% \text { Increase }
\end{aligned}
$$

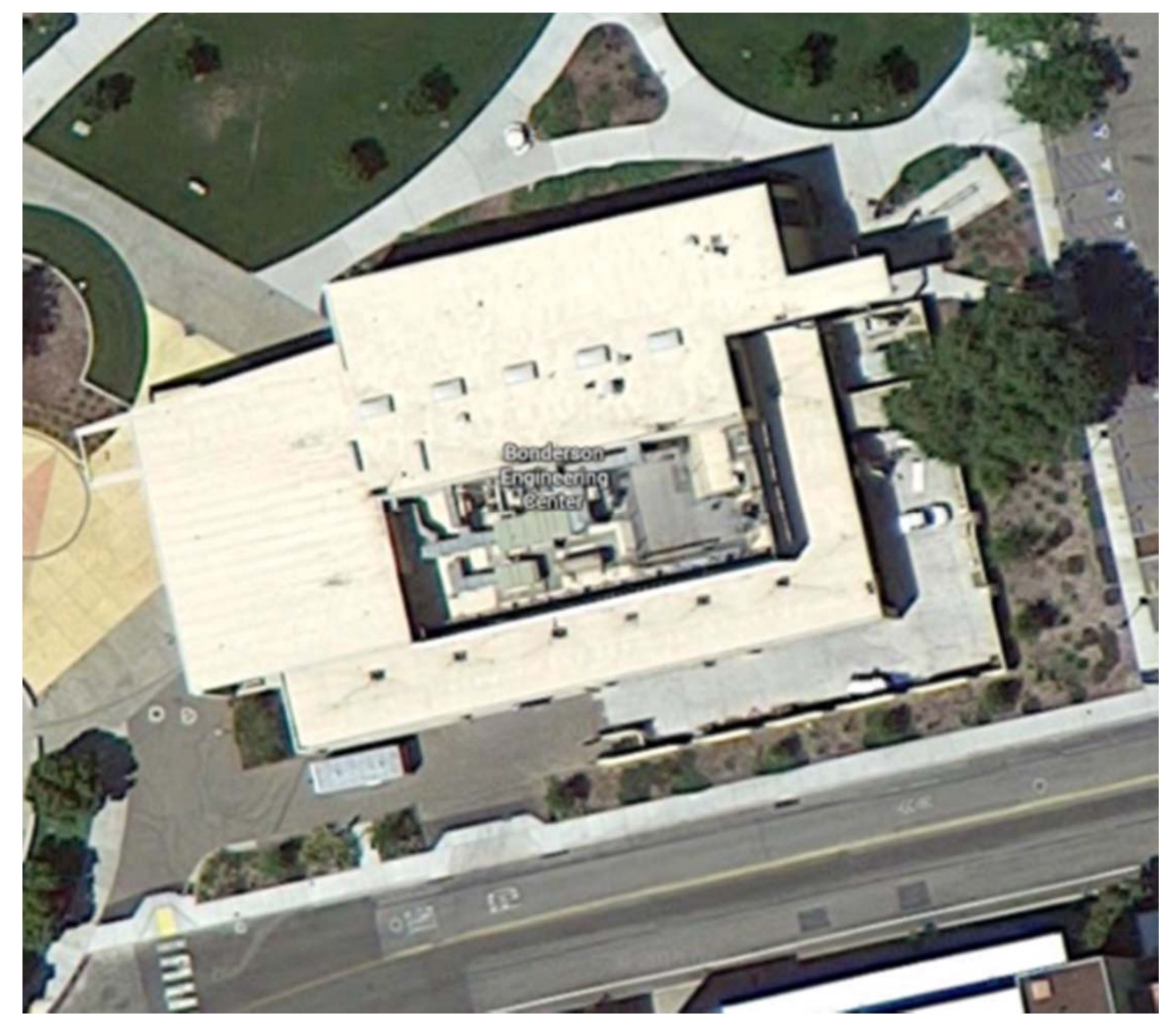




\section{Area Increases}

\begin{tabular}{|l|c|}
\hline Original Allowable Area Per Story & $9,500 \mathrm{ft}^{2}$ \\
\hline Frontage Increase & $7,125 \mathrm{ft}^{2}$ \\
\hline Automatic Sprinkler Increase & $19,000 \mathrm{ft}^{2}$ \\
\hline Allowable Area Per Story & $35,625 \mathrm{ft}^{2}$ \\
\hline
\end{tabular}

$$
\begin{aligned}
A_{a}= & \left\{A_{t}+\left[A_{t} \times I_{f}\right]+\left[A_{t} \times I_{s}\right]\right\} \quad \text { (Equation 5-1) } \\
\text { where: } & \\
A_{a}= & \text { Allowable building area per story (square feet). } \\
A_{t}= & \text { Tabular building area per story in accordance with } \\
& \text { Table } 503 \text { (square feet). } \\
I_{f}= & \begin{array}{l}
\text { Area increase factor due to frontage as calculated in } \\
\text { accordance with Section } 506.2 .
\end{array} \\
I_{s}= & \begin{array}{l}
\text { Area increase factor due to sprinkler protection as } \\
\text { calculated in accordance with Section 506.3. }
\end{array}
\end{aligned}
$$

- $1^{\text {st }}$ Floor Area: $12,240 \mathrm{ft}^{2}$

- Allowable building height and story increase due to automatic sprinkler system installation is not needed. 


\section{Fire Suppression System}

- Fully Sprinklered

- Wet-Pipe System

- Quick Response Sprinklers (K-Factor 5.6, 200 F Activation Temperature)

- 270 Total Sprinklers

- Light Hazard (Public Areas, Offices, Classrooms)

- Ordinary Hazard 1 (Storage, Equipment, Maintenance, Vocational Rooms, School Shop)

- No Fire Pump 


\section{Fire Suppression Design}

\begin{tabular}{|c|c|c|c|c|c|}
\hline Classification & Density $\left(\mathrm{gpm} / \mathrm{ft}^{2}\right)$ & $\begin{array}{c}\text { Original Area of } \\
\text { Sprinkler Operation }\left(\mathrm{ft}^{2}\right)\end{array}$ & $\begin{array}{c}\text { *Reduced Area of } \\
\text { Sprinkler Operation }\left(\mathrm{ft}^{2}\right)\end{array}$ & $\begin{array}{c}\text { Hose Stream } \\
\text { Allowance }(\mathrm{gpm})\end{array}$ & Duration (minutes) \\
\hline Light Hazard & 0.1 & 1500 & 990 & 100 & 30 \\
\hline Ordinard Hazard I & 0.15 & 1500 & 990 & 250 & 60 \\
\hline
\end{tabular}

*Area of Sprinkler Operation permited to be reduced without revising density per NFPA 13 11.2.3.2.3.1

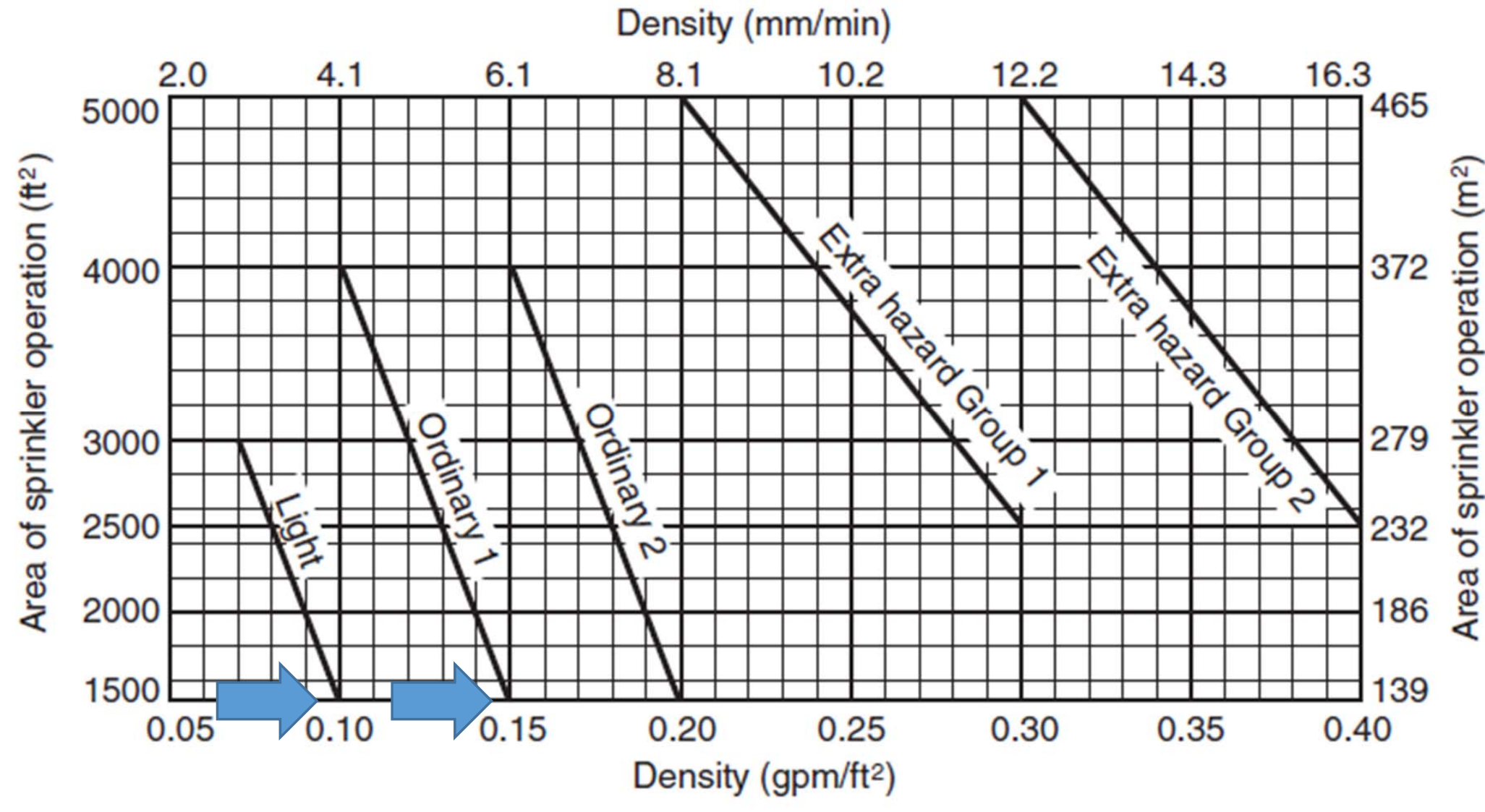

CAL POLY

Fire Protection Engineering 


\section{Reduction Calculation}

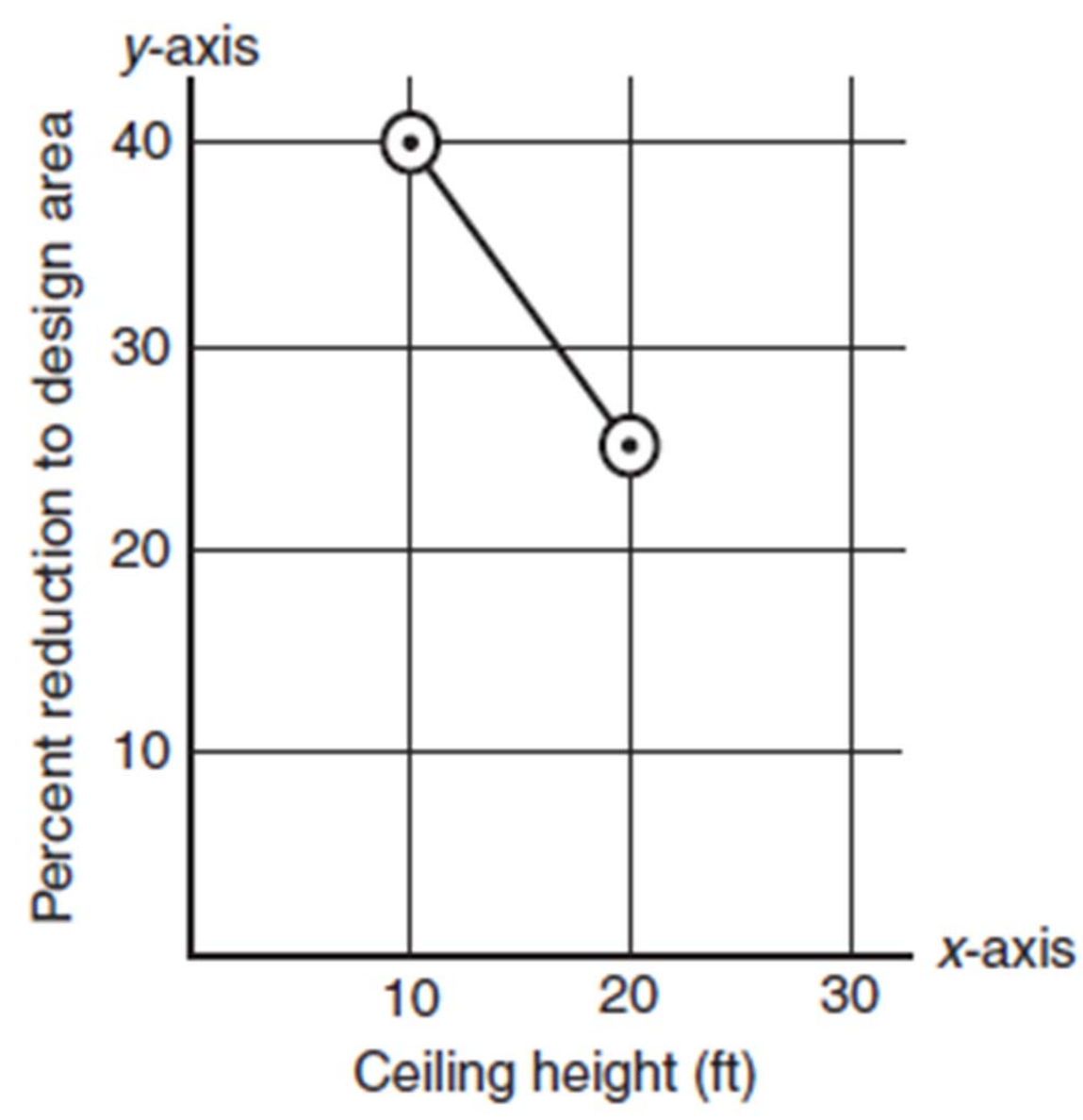

$$
\begin{gathered}
\text { Ceiling Height }=14^{\prime} 1^{\prime \prime} \\
Y=\frac{-3\left(14^{\prime} 1^{\prime \prime}\right)}{2}+55 \\
Y=33.875 \\
\text { New Area }=1500 f t^{2} X(100 \%-33.875 \%) \\
\text { New Area }=992 \mathrm{ft}^{2}
\end{gathered}
$$

Note: $y=\frac{-3 x}{2}+55$ 


\section{Remote Area}

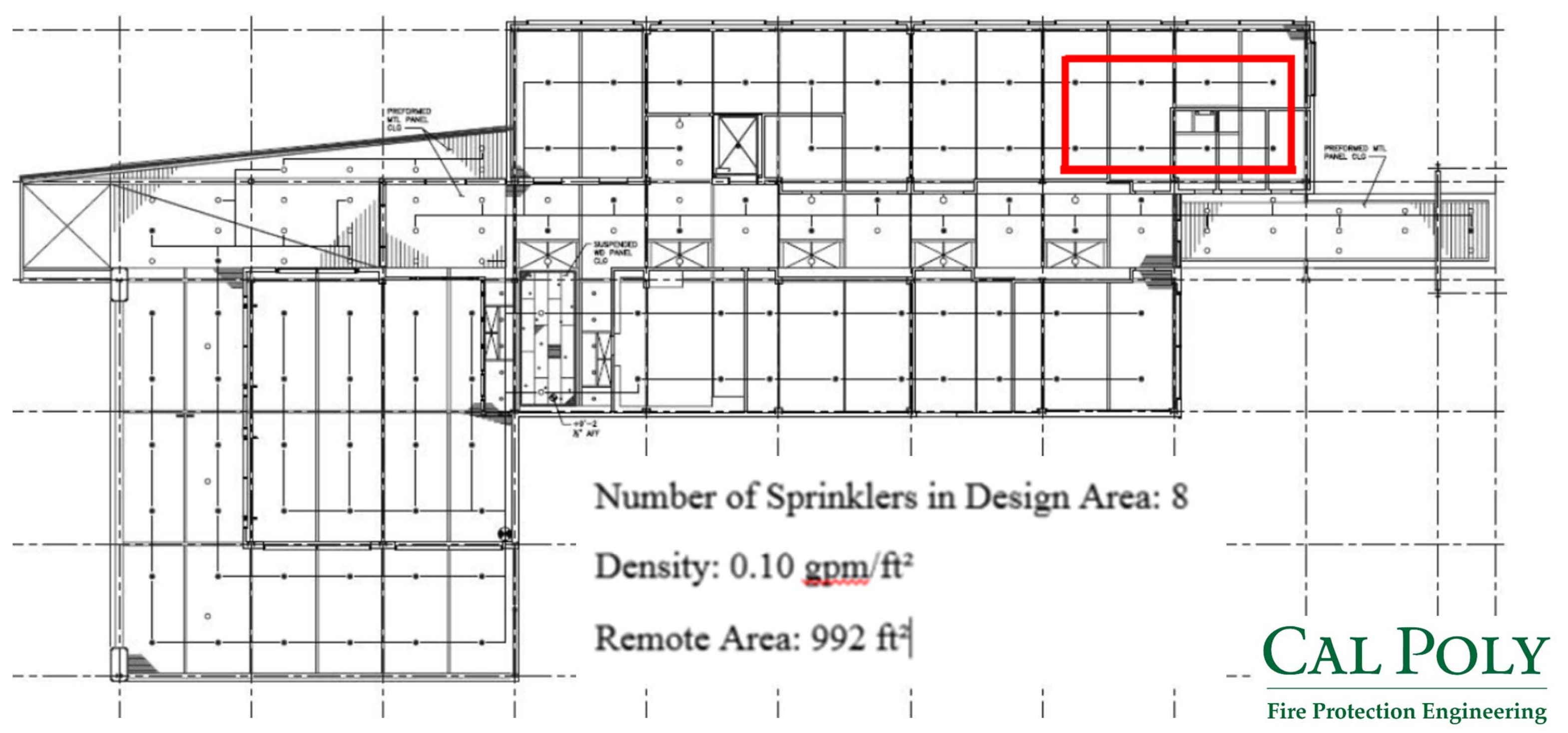




\section{Hand Calculations}

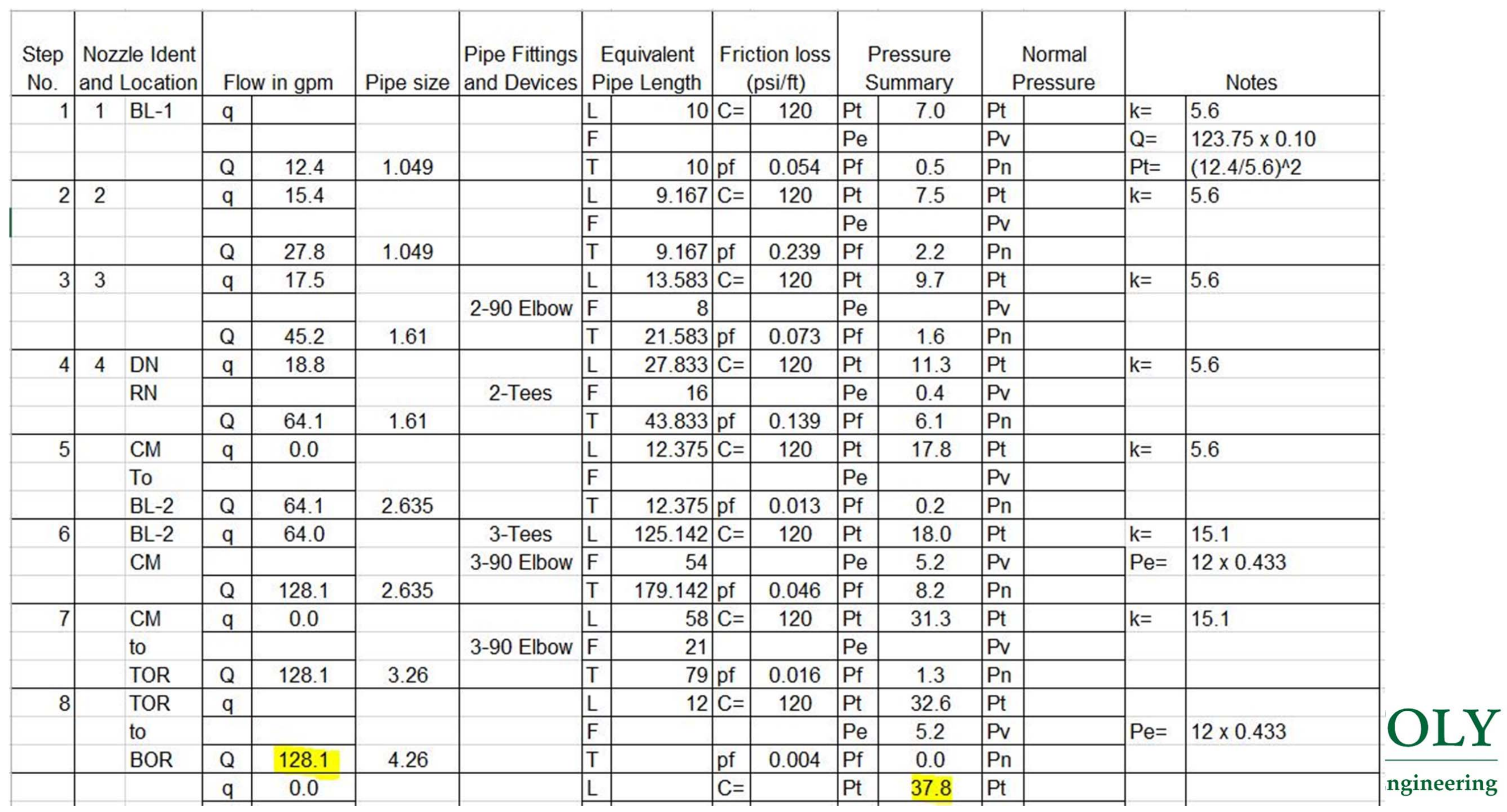




\section{Flow Test Summary Sheet}

CONTRACT NAME:Bonderson Engineering Projects Lenter no:

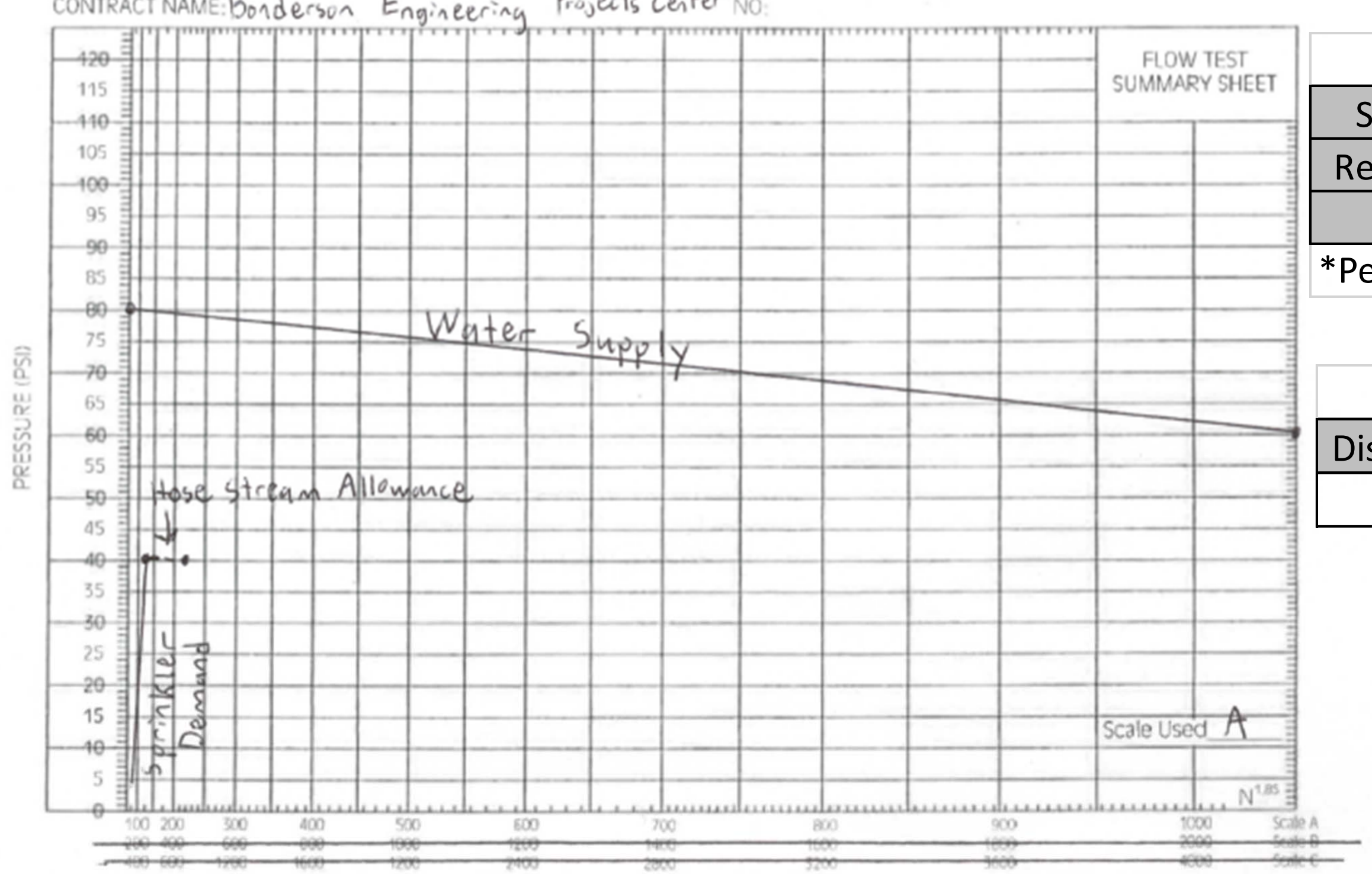

FLOW-CPM
Water Supply

\begin{tabular}{|c|c|}
\hline Static Pressure & $80 \mathrm{psi}$ \\
\hline Residual Pressure & $60 \mathrm{psi}$ \\
\hline Flow & $1210 \mathrm{gpm}$ \\
\hline
\end{tabular}

*Performed 09/03/02

System Demand

Discharge Rate

$128.1 \mathrm{gpm}$

Pressure

37.8 psi

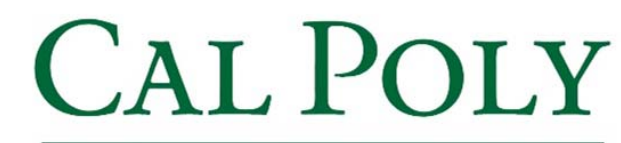

Fire Protection Engineering 


\section{Fire Detection and Alarm}

- Automatic and Manual Fire Alarm System

- Elevator Recall/Shunt

- Duct Detector Monitoring

- ADA Compliant Notification

- Fire Alarm Control Panel (FACP) located on first floor

- FACP is Notifier model NFS-640 (pictured)

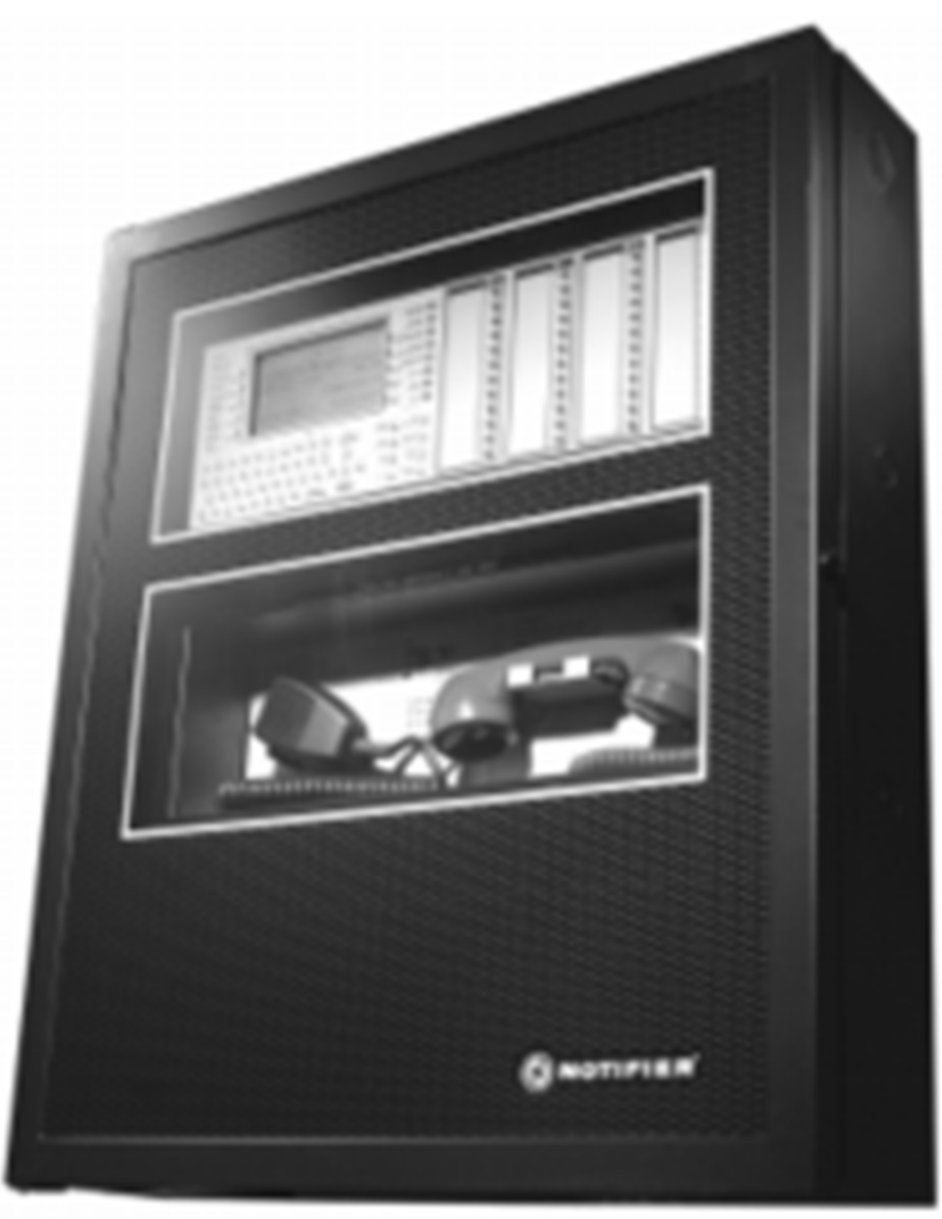

- No Mass Notification System 


\section{Detection Devices}
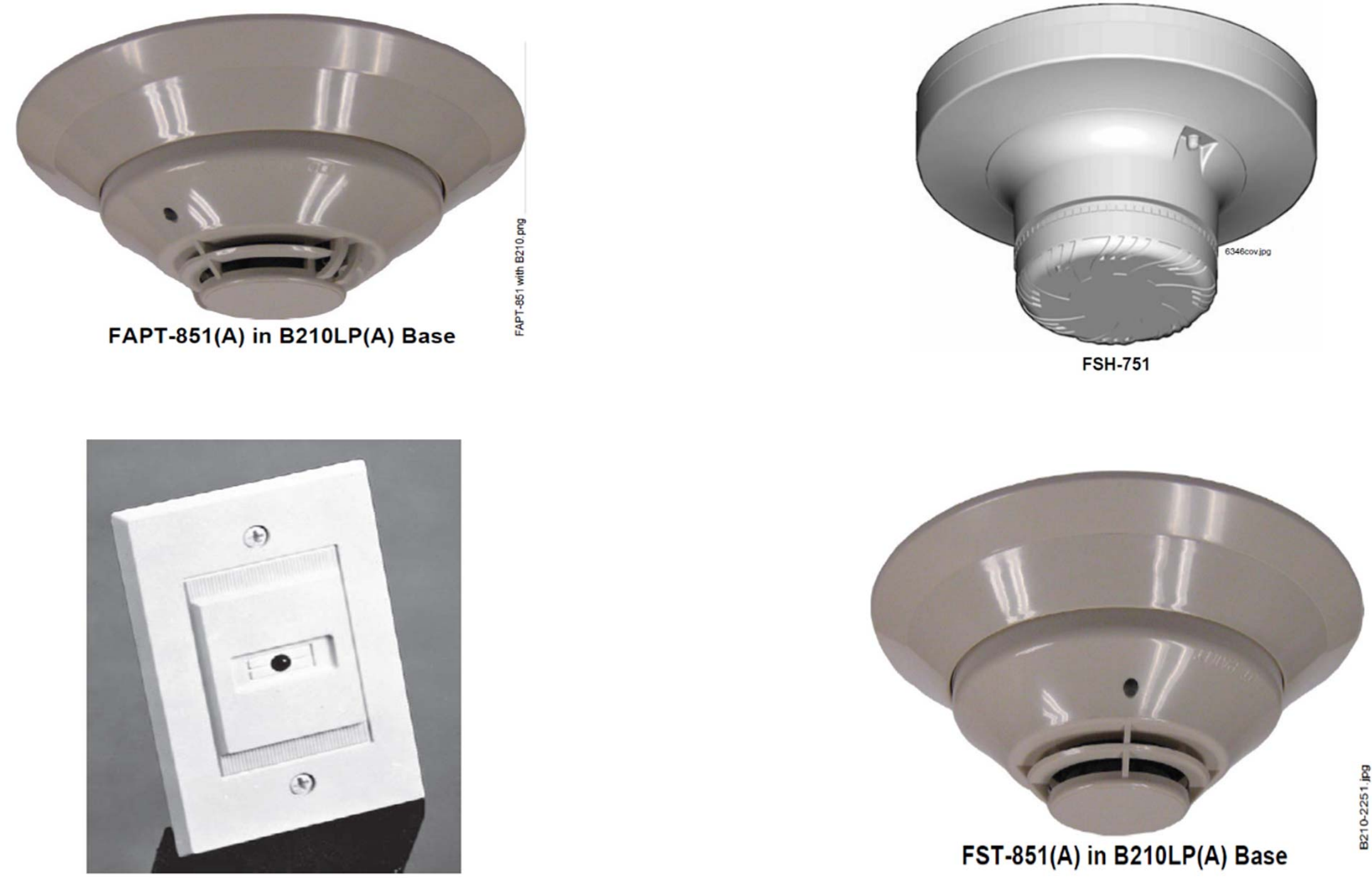

NOTIFIER FSD-751P 


\section{$1^{\text {st }}$ Floor}

FACP
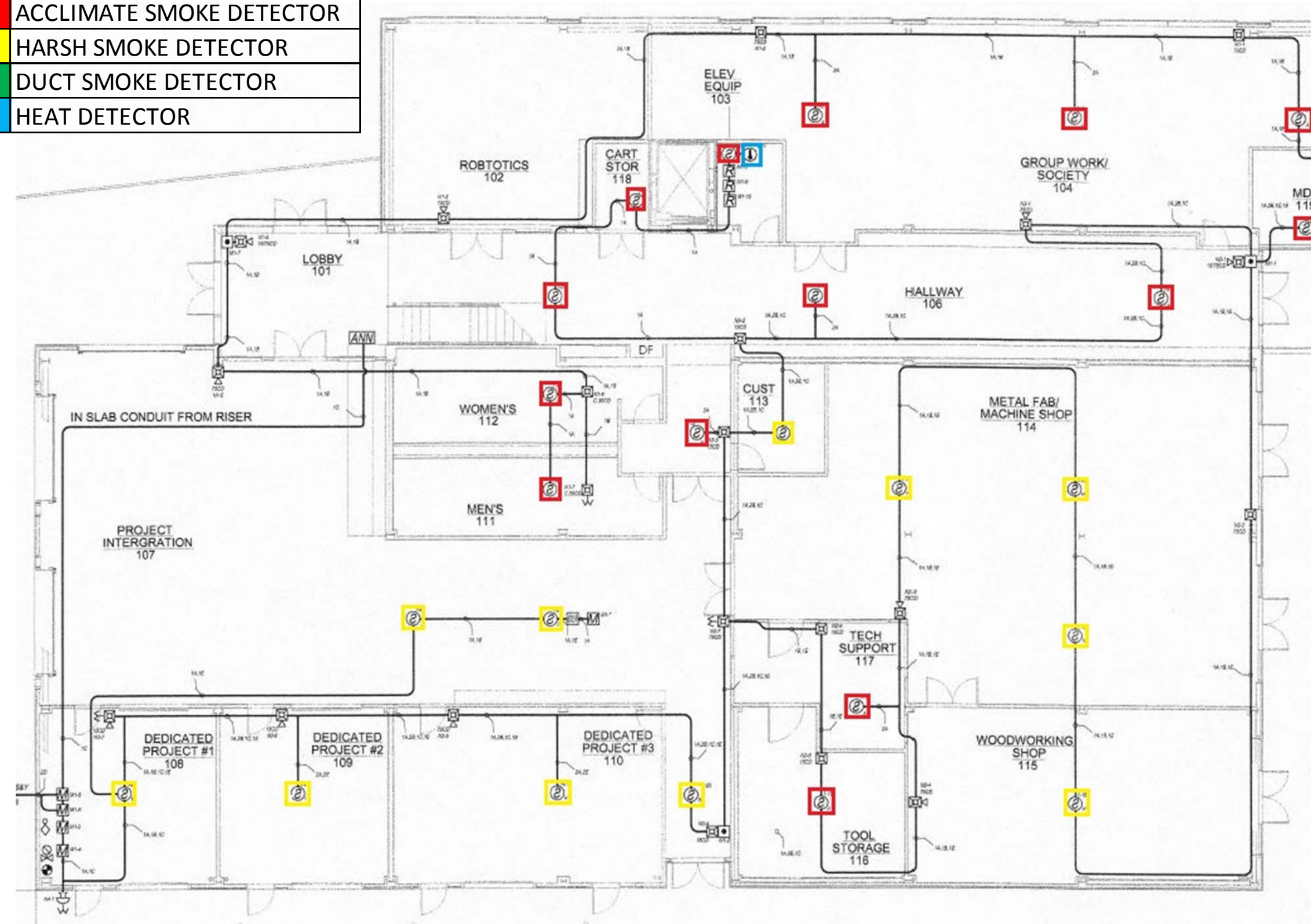


\section{$2^{\text {nd }}$ Floor}

ACCLIMATE SMOKE DETECTOR

HARSH SMOKE DETECTOR

DUCT SMOKE DETECTOR

HEAT DETECTOR

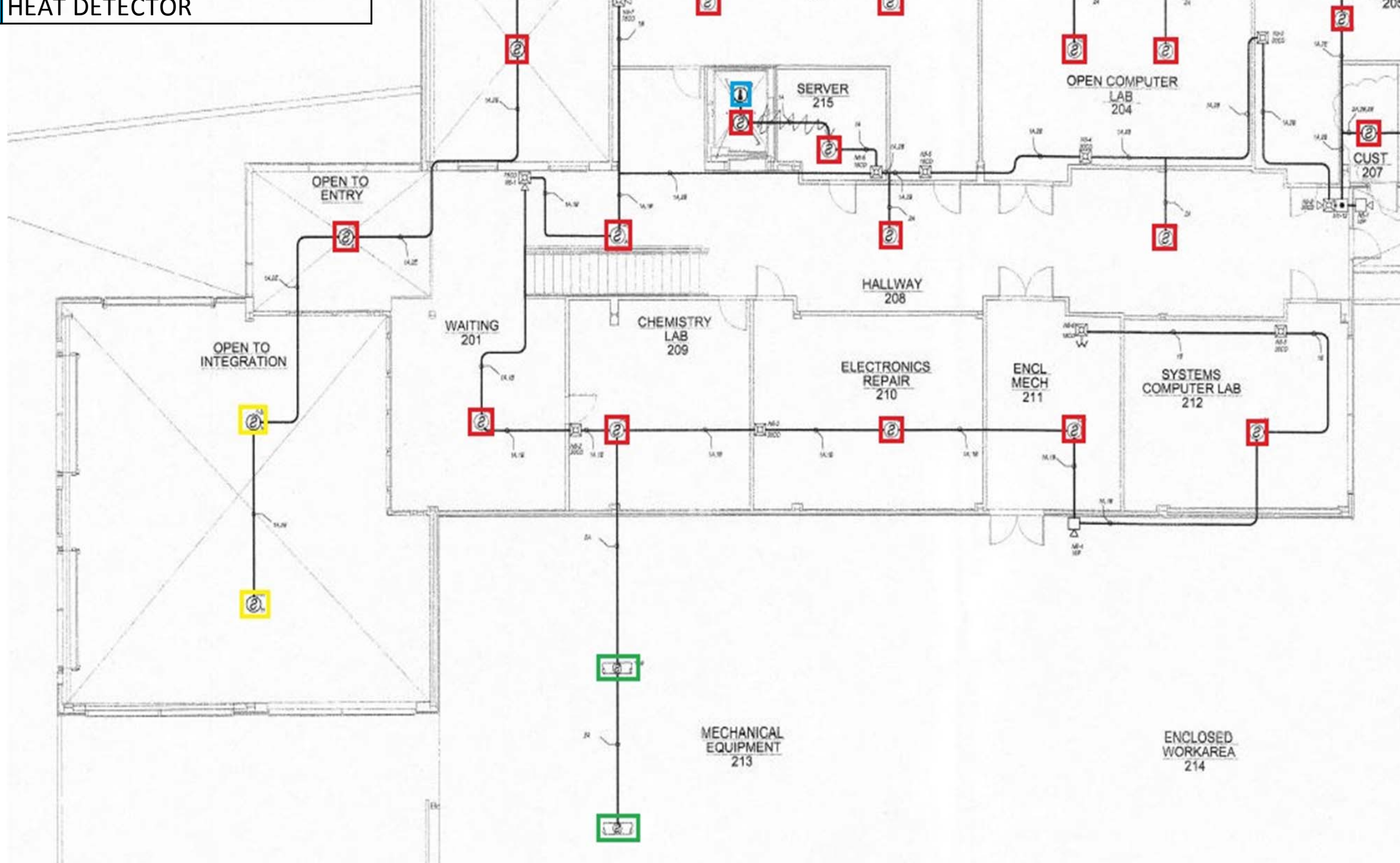




\section{Performance Based Design}

- Two Fire Scenarios:

1. Corridor Fire

2. Project Integration Room

- Used Fire Dynamics Simulator to

evaluate tenability requirements (ASET)

- Used Pathfinder to determine RSET

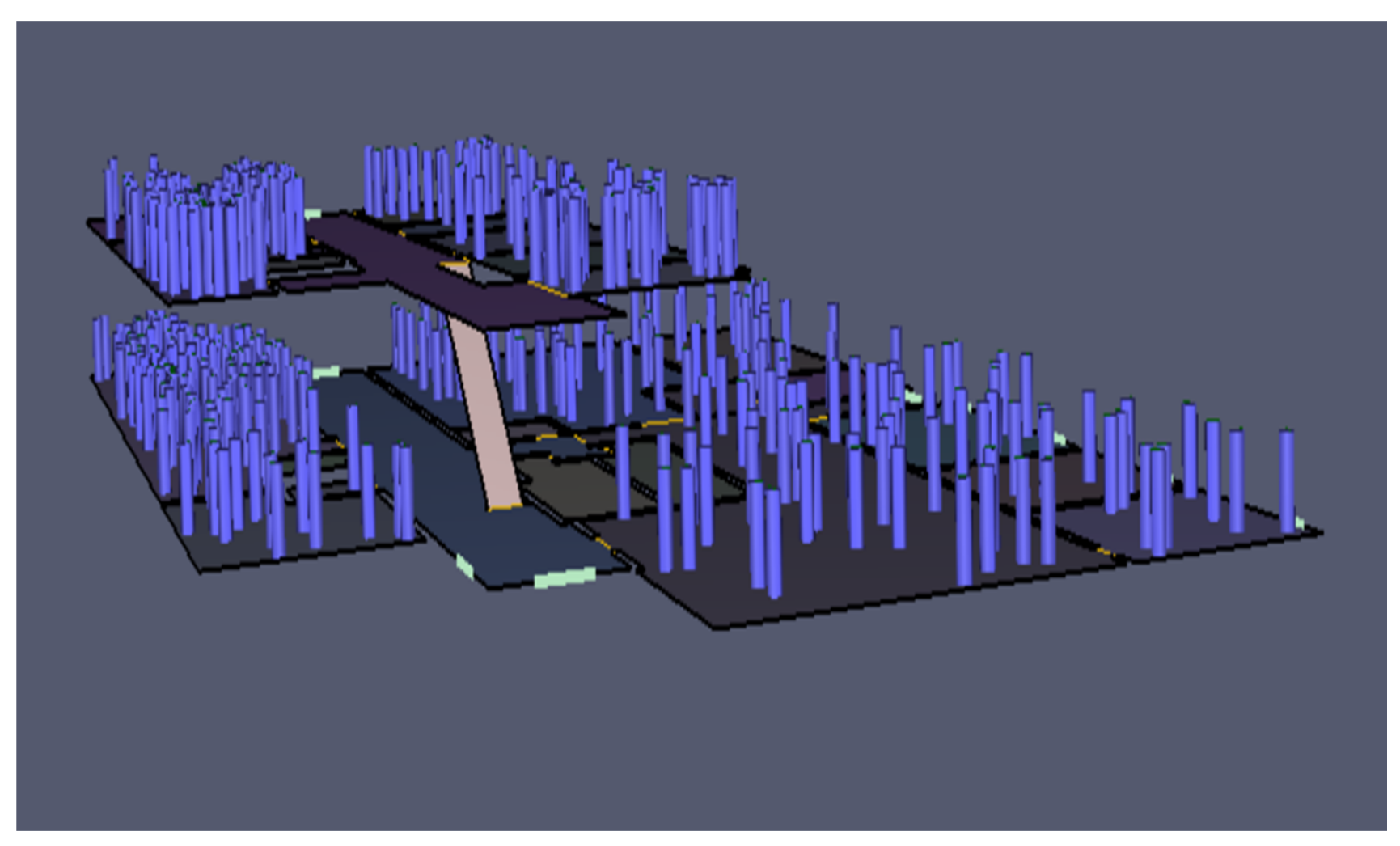




\section{ASET VS RSET}

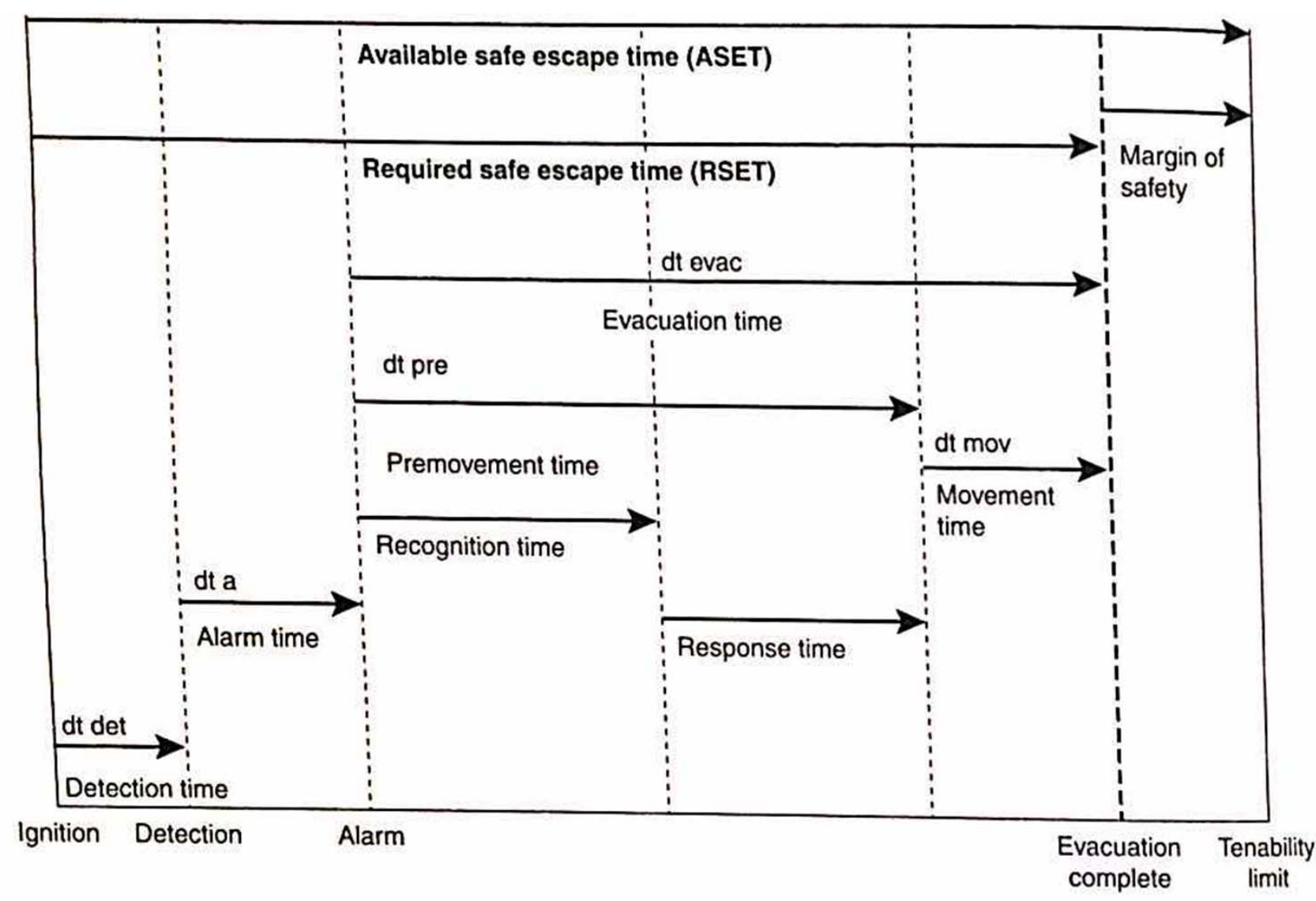

RSET $=t_{d}+t_{n}+t_{p-e}\left(s_{f}\right)+t_{e}\left(s_{f}\right)$

RSET = Total Evacuation Time

$t_{d}=$ Time from fire ignition to detection

$t_{n}=$ Time from detection to notification of occupants of a fire emergency

$t_{p-e}=$ Time from notification until evacuation commences

$t_{e}=$ Time from the start of evacuation until it is completed

$s_{f}=$ Safety factor

Figure 3-12.1. Egress time model. 


\section{RSET}

$R S E T=t_{d}+t_{n}+t_{p-e}\left(s_{f}\right)+t_{e}\left(s_{f}\right)$

RSET =Total Evacuation Time

$\mathrm{t}_{\mathrm{d}}=9.6$ seconds

$t_{n}=10$ seconds

$t_{p-e}=$ Time from notification until evacuation commences

$t_{e}=$ Time from the start of evacuation until it is completed

$s_{f}=$ Safety factor

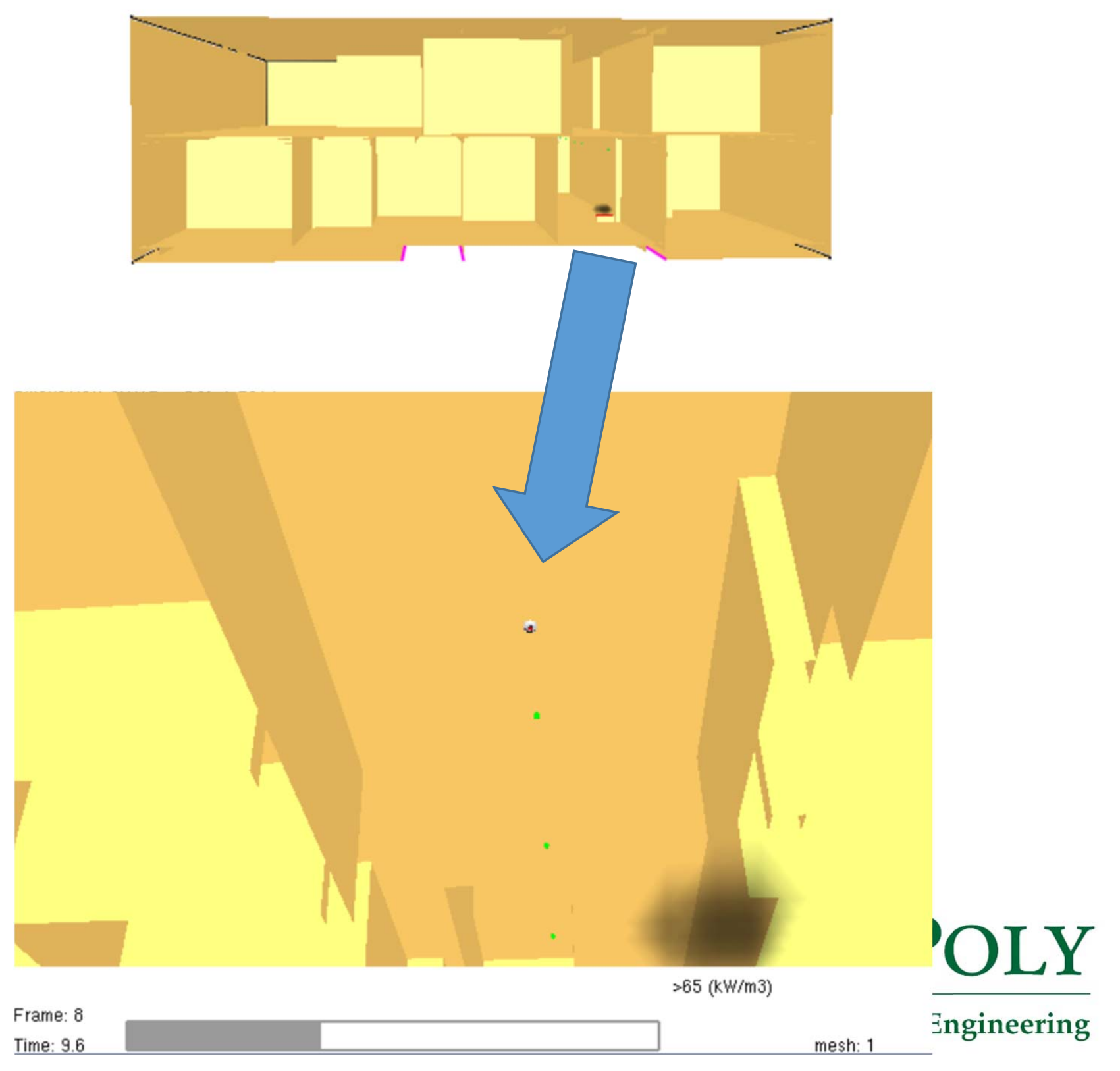




\section{RSET}

$R S E T=t_{d}+t_{n}+t_{p-e}\left(\mathrm{~s}_{f}\right)+t_{e}\left(\mathrm{~s}_{f}\right)$

RSET =Total Evacuation Time

$\mathrm{t}_{\mathrm{d}}=9.6$ seconds

$t_{n}=10$ seconds

$t_{p-e}=0$ seconds

$t_{e}=$ Time from the start of evacuation until it is completed

$s_{f}=$ Safety factor

Table 3-12.3 Design Behavioral Scenarios and Occupancy Types ${ }^{4}$

\begin{tabular}{|c|c|c|c|c|c|}
\hline Category & Occupant Alertness & $\begin{array}{l}\text { Occupant } \\
\text { Familiarity }\end{array}$ & $\begin{array}{l}\text { Occupant } \\
\text { Density }\end{array}$ & $\begin{array}{l}\text { Enclosure/ } \\
\text { Complexity }\end{array}$ & $\begin{array}{c}\text { Examples of Occupancy } \\
\text { Types }\end{array}$ \\
\hline A & Awake & Familiar & Low & One or many & Office or industrial \\
\hline B1 & Awake & Unfamiliar & High & One or few & $\begin{array}{l}\text { Shop, restaurant, circulation } \\
\text { space }\end{array}$ \\
\hline \multirow[t]{2}{*}{ B2 } & Awake & Unfamiliar & High & $\begin{array}{l}\text { One with } \\
\text { focal point }\end{array}$ & Cinema, theatre \\
\hline & Asleep & Familiar & Low & Few & Dwelling \\
\hline $\mathrm{Ci}$ & $\begin{array}{l}\text { Long term, individual } \\
\text { occupancy }\end{array}$ & & & & $\begin{array}{l}\text { Without } 24 \mathrm{hr} \text { on-site } \\
\text { management }\end{array}$ \\
\hline Cii & Managed occupancy & & & & $\begin{array}{l}\text { Serviced flats, halls of } \\
\text { residence, etc. }\end{array}$ \\
\hline Ciii & Asleep & Unfamiliar & Low & Many & Hotel, hostel \\
\hline$\underline{\mathrm{D}}$ & Medical care & Unfamiliar & Low & Many & Residential (institutional) \\
\hline E & Transportation & Unfamiliar & High & Many & Railway station/airport \\
\hline
\end{tabular}




\section{RSET-Pathfinder}

\section{First Floor}

$R S E T=t_{d}+t_{n}+t_{p-e}\left(s_{f}\right)+t_{e}\left(s_{f}\right)$

RSET =Total Evacuation Time

$t_{d}=9.6$ seconds

$t_{n}=10$ seconds

$t_{p-e}=0$ seconds

$t_{e}=76.4$ seconds

$s_{f}=$ Safety factor

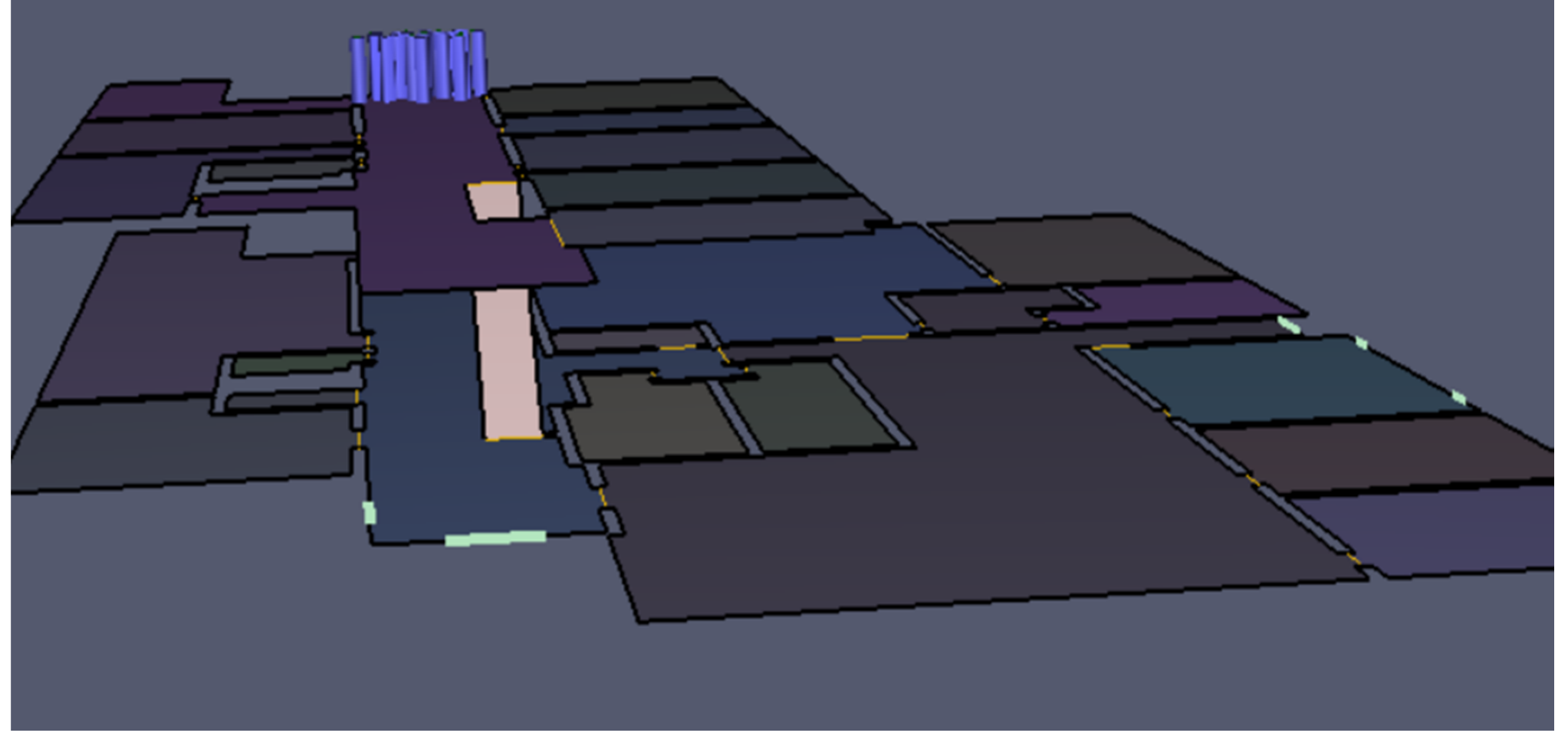




\section{RSET-Pathfinder}

\section{Second Floor}

$R S E T=t_{d}+t_{n}+t_{p-e}\left(s_{f}\right)+t_{e}\left(s_{f}\right)$

RSET =Total Evacuation Time

$t_{d}=9.6$ seconds

$t_{n}=10$ seconds

$t_{p-e}=0$ seconds

$t_{e}=94.3$ seconds

$s_{f}=$ Safety factor

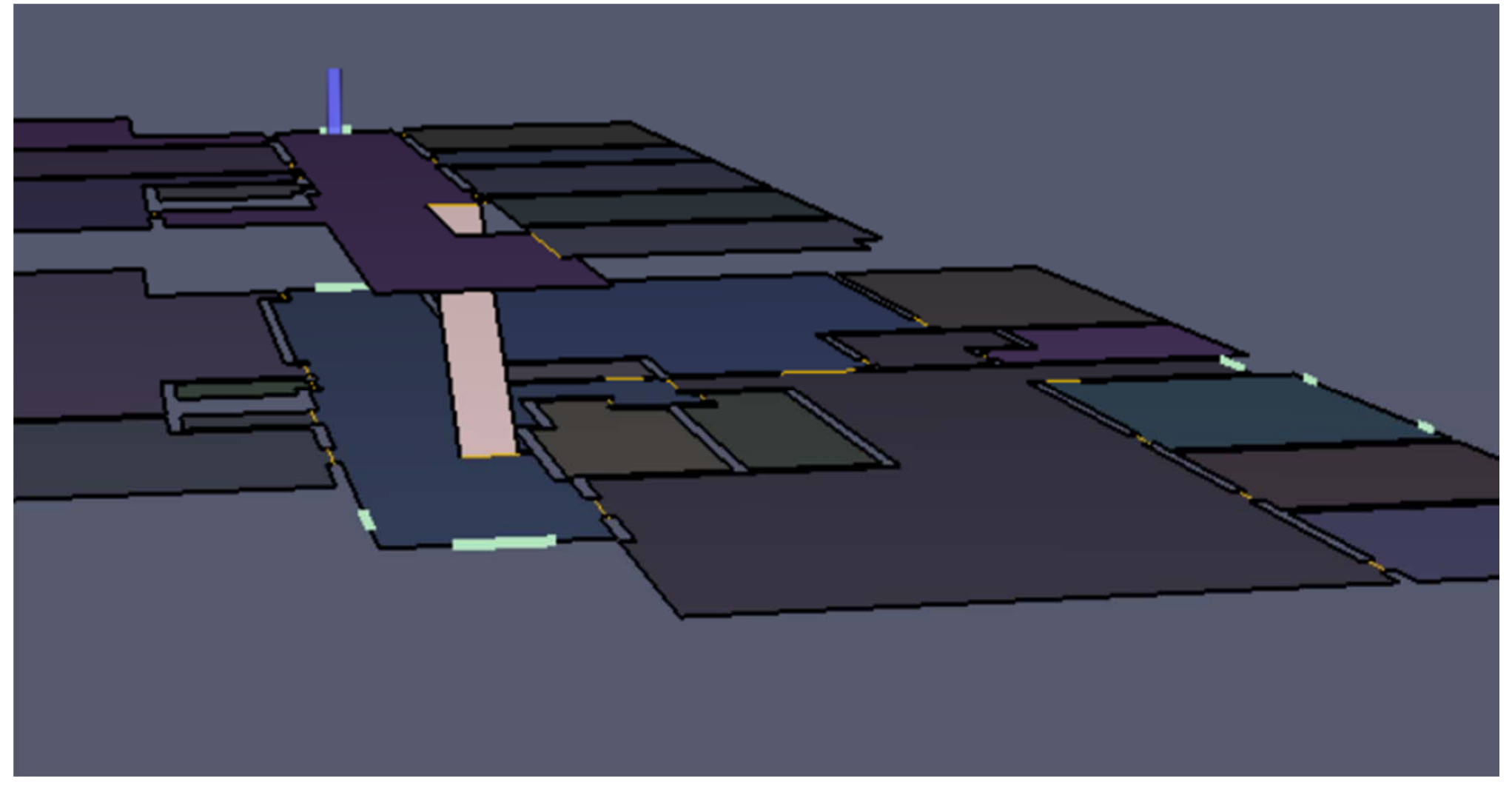




\section{Detection Time}

$R S E T=t_{d}+t_{n}+t_{p-e}\left(s_{f}\right)+t_{e}\left(s_{f}\right)$

RSET = Total Evacuation Time

$\mathrm{t}_{\mathrm{d}}=9.6$ seconds

$t_{n}=10$ seconds

$t_{p-e}=0$ seconds

$t_{e}=76.4$ and 94.3 seconds

$s_{f}=1.5$
Total RSET First Floor $=135$ Seconds Total RSET Second Floor $=161$ Seconds

RSET is rounded to 3 minutes for tenability equations 


\section{ASET-Visibility}

- Measured at 6 feet above walking surface on each level

- Case could be made to reduce to 4 meters for occupants familiar with their escape route for small enclosures

\begin{tabular}{|l|l|l|}
\hline \multicolumn{1}{|c|}{ Tenability Criteria } & \multicolumn{1}{c|}{ Metric Units } & \multicolumn{1}{c|}{ Imperial Units } \\
\hline Visibility & 10 meters & 33 feet \\
\hline Temperature & $133^{\circ} \mathrm{C}$ & $272^{\circ} \mathrm{F}$ \\
\hline Toxicity (CO) & $8,000 \mathrm{ppm}$ & $8,000 \mathrm{ppm}$ \\
\hline
\end{tabular}

- $30 \%$ of people turn back rather than enter a room at 4 meters 


\section{ASET-Temperature}

$t=\left(5 \times 10^{7}\right)\left(T^{-3.4}\right)$

Where: $t=$ time to incapacitation $(\min )$

$\mathrm{T}=$ temperature $\left({ }^{\circ} \mathrm{C}\right)$

Rearranging:

$T=\sqrt[3.4]{5 \times 10^{7} / 3}$

\begin{tabular}{|l|l|l|}
\hline \multicolumn{1}{|c|}{ Tenability Criteria } & Metric Units & \multicolumn{1}{c|}{ Imperial Units } \\
\hline Visibility & 10 meters & 33 feet \\
\hline Temperature & $133^{\circ} \mathrm{C}$ & $272^{\circ} \mathrm{F}$ \\
\hline Toxicity (CO) & $8,000 \mathrm{ppm}$ & $8,000 \mathrm{ppm}$ \\
\hline
\end{tabular}

$\mathrm{T}=133^{\circ} \mathrm{C}$ 


\section{ASET-Toxicity (CO)}

$\% \mathrm{COHb}=\left(3.317 \times 10^{-5}\right) \times(\mathrm{ppm} \mathrm{CO})^{1.036} \times(\mathrm{RMV}) \times(\mathrm{t})$

Where: $\% \mathrm{COHb}=$ Percentage of $\mathrm{COHb}$ in the blood ppm $\mathrm{CO}=\mathrm{CO}$ concentration in the air

$\mathrm{RMV}=\mathbf{2 5} \mathrm{L} / \mathrm{min}$ for a person doing light work (such as walking)

$t=$ exposure time $(\min )=3$ minutes

Rearranging:

\begin{tabular}{|l|c|c|}
\hline \multicolumn{1}{|c|}{$\begin{array}{c}\text { Activity Level of } \\
\text { Subject }\end{array}$} & $\mathbf{V}(\mathbf{L} / \mathbf{m i n})$ & $\mathbf{D}$ (percent COHb) \\
\hline $\begin{array}{l}\text { Resting or sleeping } \\
\text { Light work- walking to } \\
\text { escape }\end{array}$ & 8.5 & 40 \\
\hline $\begin{array}{l}\text { Heavy work- slow } \\
\text { running, walking up } \\
\text { stairs }\end{array}$ & 25 & 30 \\
\hline
\end{tabular}

ppm CO $=\left[\% \mathrm{COHb} /\left\{\left(3.317 \times 10^{-5}\right) \times(\mathrm{RMV}) \times(\mathrm{t})\right\}\right]^{0.965}$

ppm CO $=\left[30 /\left\{\left(3.317 \times 10^{-5}\right) \times(25) \times(3)\right\}\right]^{0.965}$

CO Concentration Criterion for a 3 Minute Exposure $=8,680 \mathrm{ppm}$ 


\section{ASET-Toxicity (CO)}

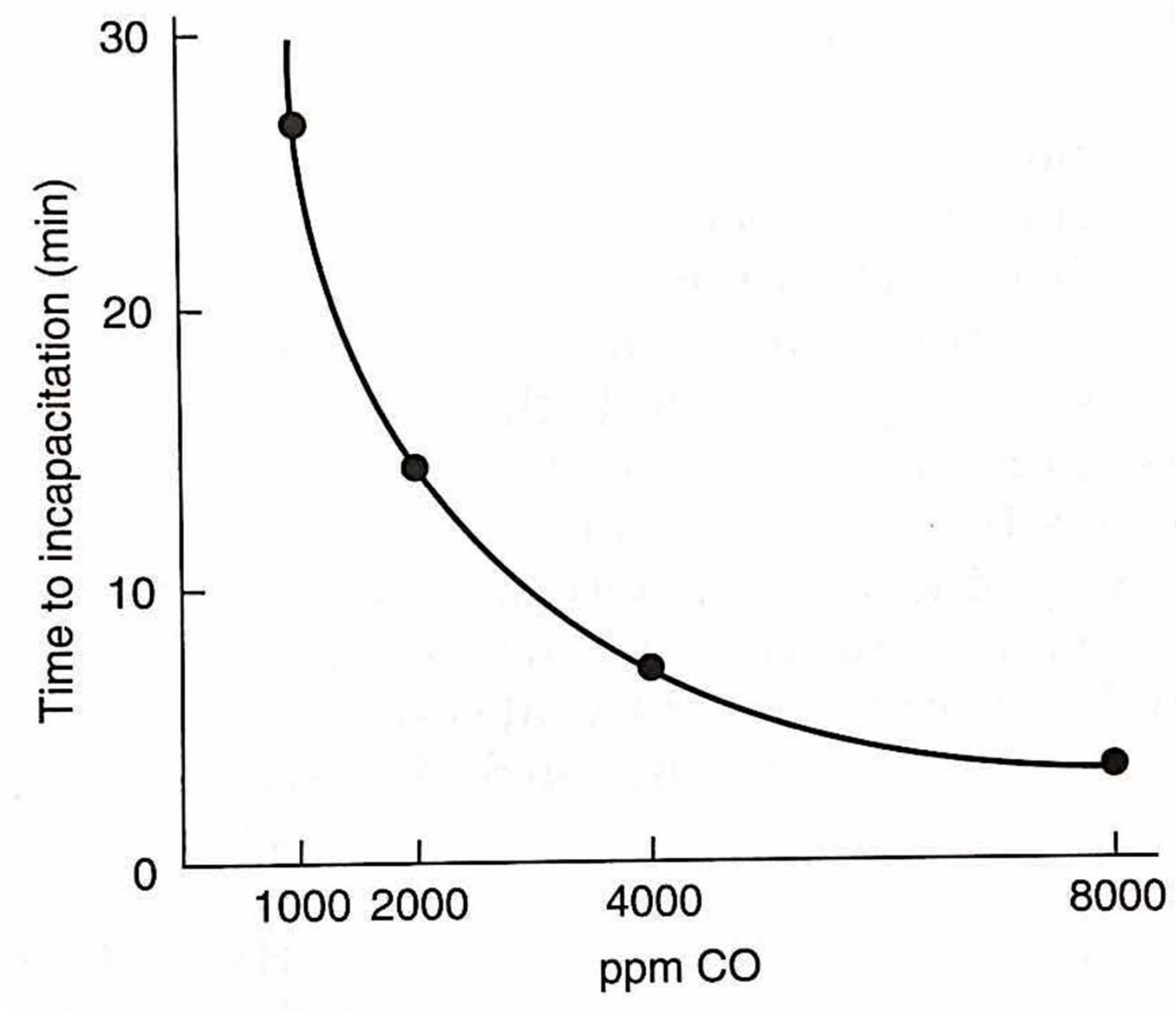

\begin{tabular}{|l|l|l|}
\hline \multicolumn{1}{|c|}{ Tenability Criteria } & \multicolumn{1}{c|}{ Metric Units } & \multicolumn{1}{c|}{ Imperial Units } \\
\hline Visibility & 10 meters & 33 feet \\
\hline Temperature & $133^{\circ} \mathrm{C}$ & $272^{\circ} \mathrm{F}$ \\
\hline Toxicity (CO) & $8,000 \mathrm{ppm}$ & $8,000 \mathrm{ppm}$ \\
\hline
\end{tabular}




\section{Design Fire 1- Corridor Fire}
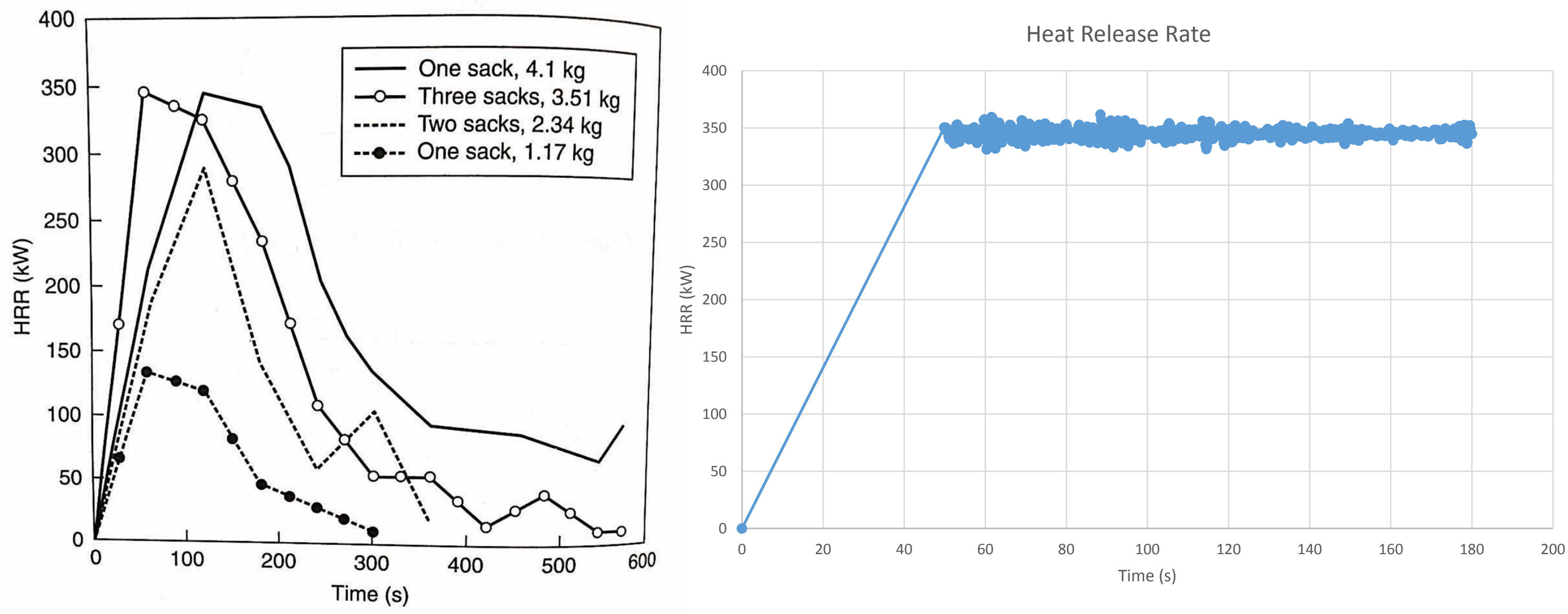

Figure 3-1.96. HRR of trash bags. 


\section{Visibility- First Floor}

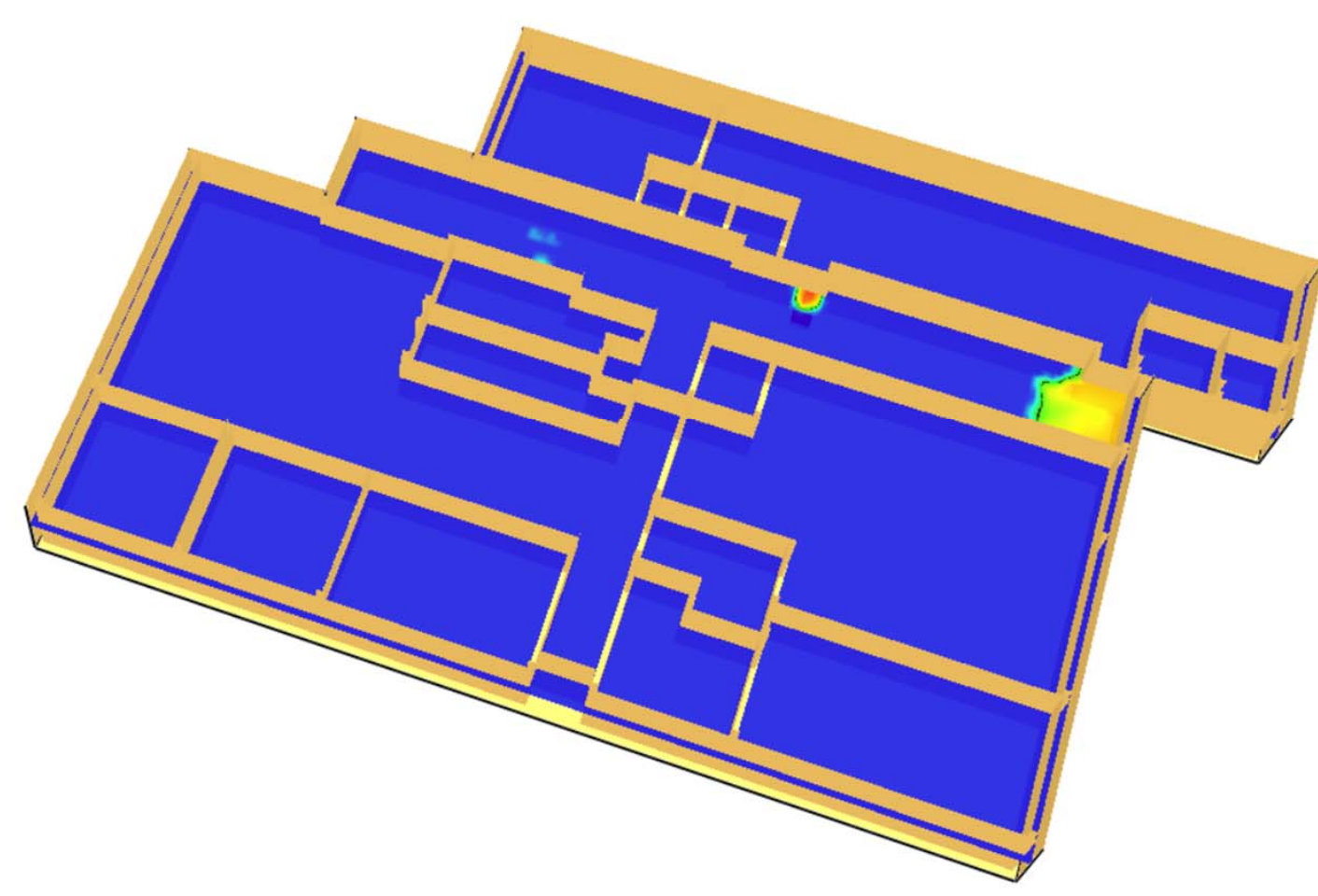

30 Seconds

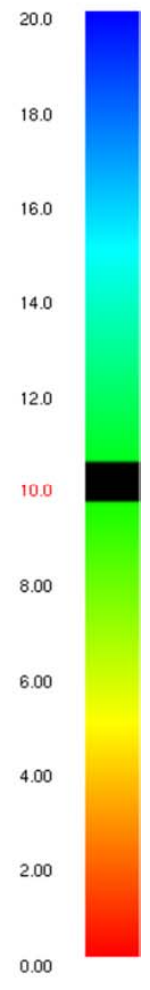

135 Seconds

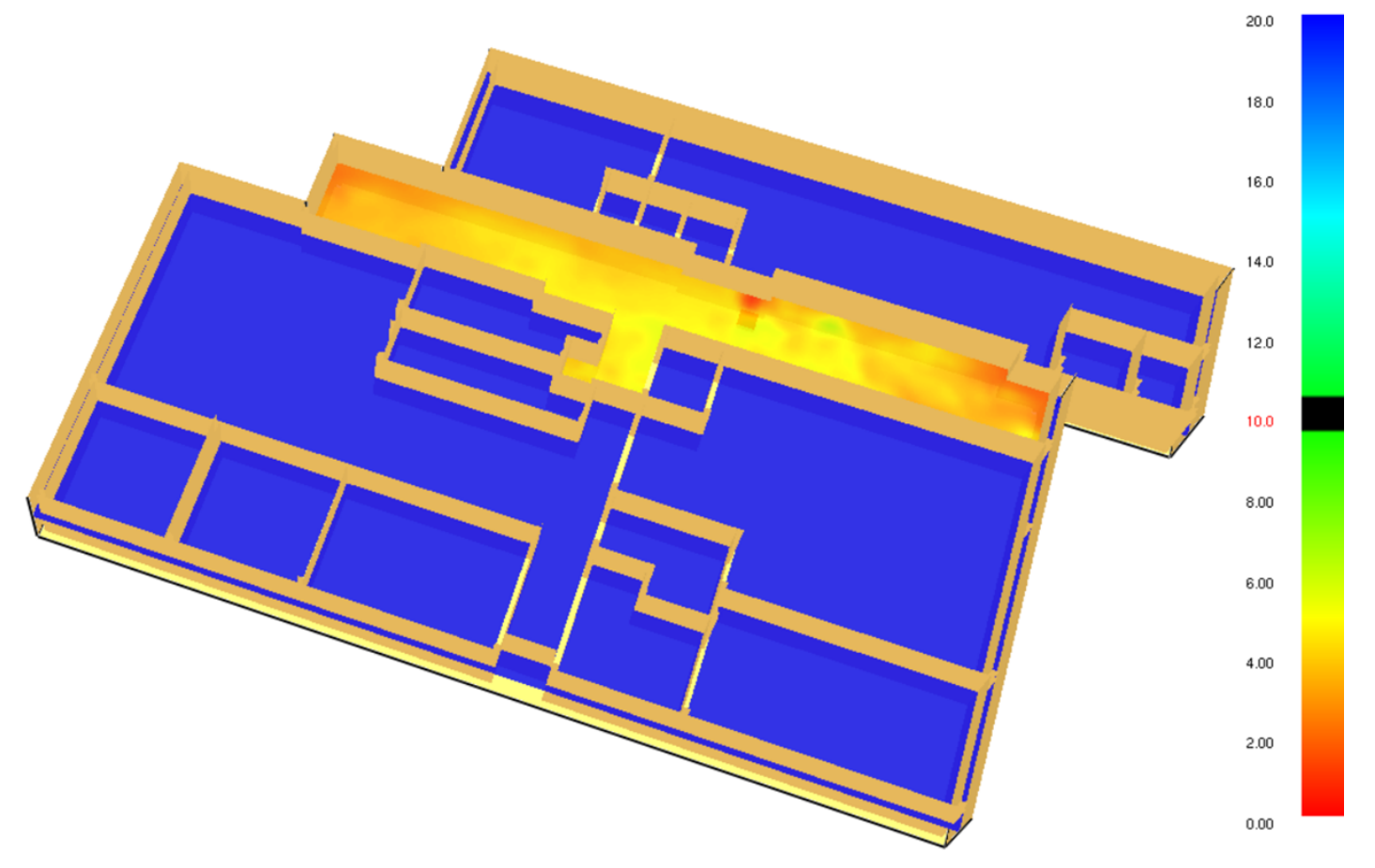

Cal Poly

Fire Protection Engineering 


\section{Visibility- Second Floor}

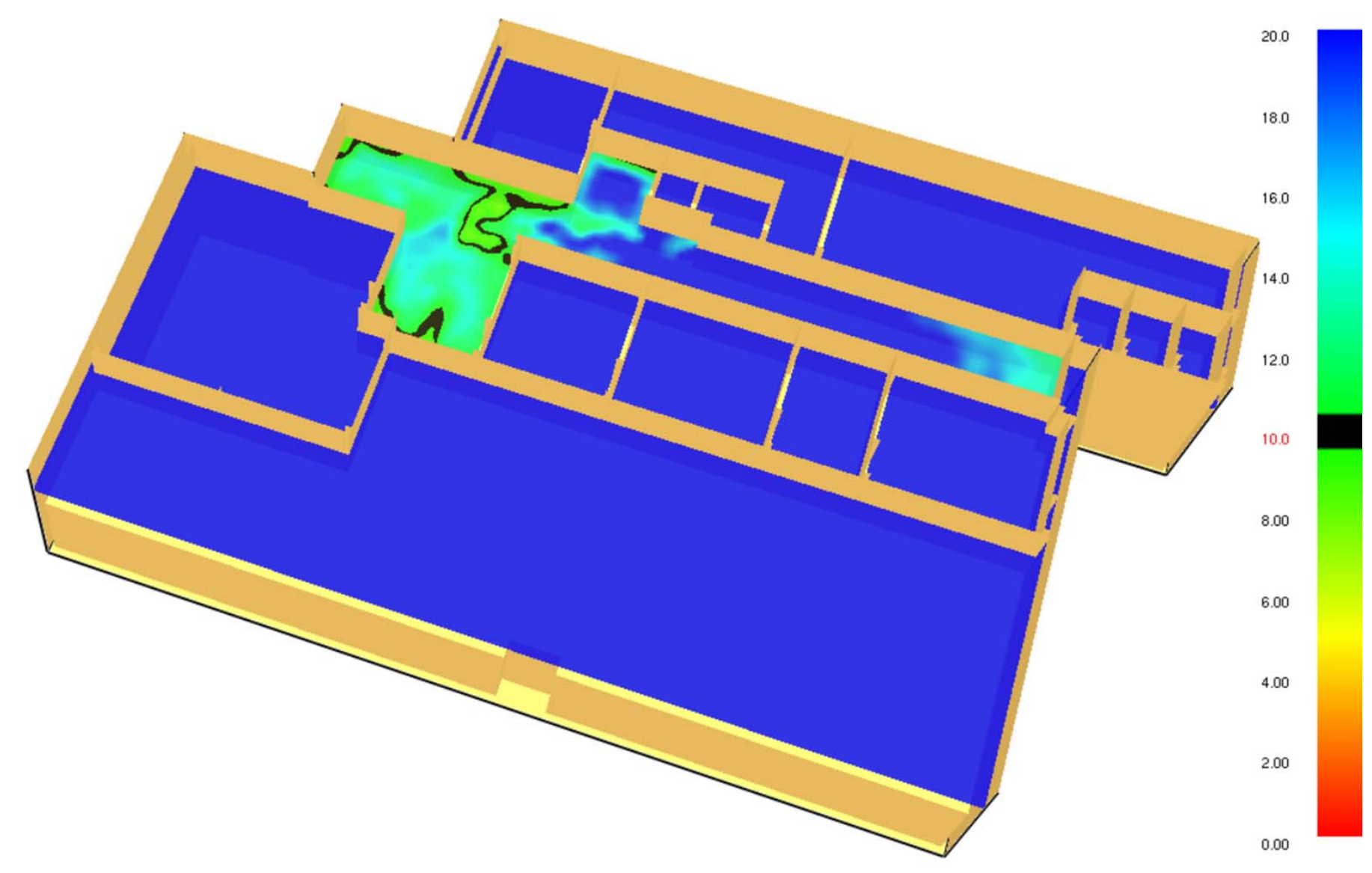

161 Seconds 


\section{Temperature- First Floor}

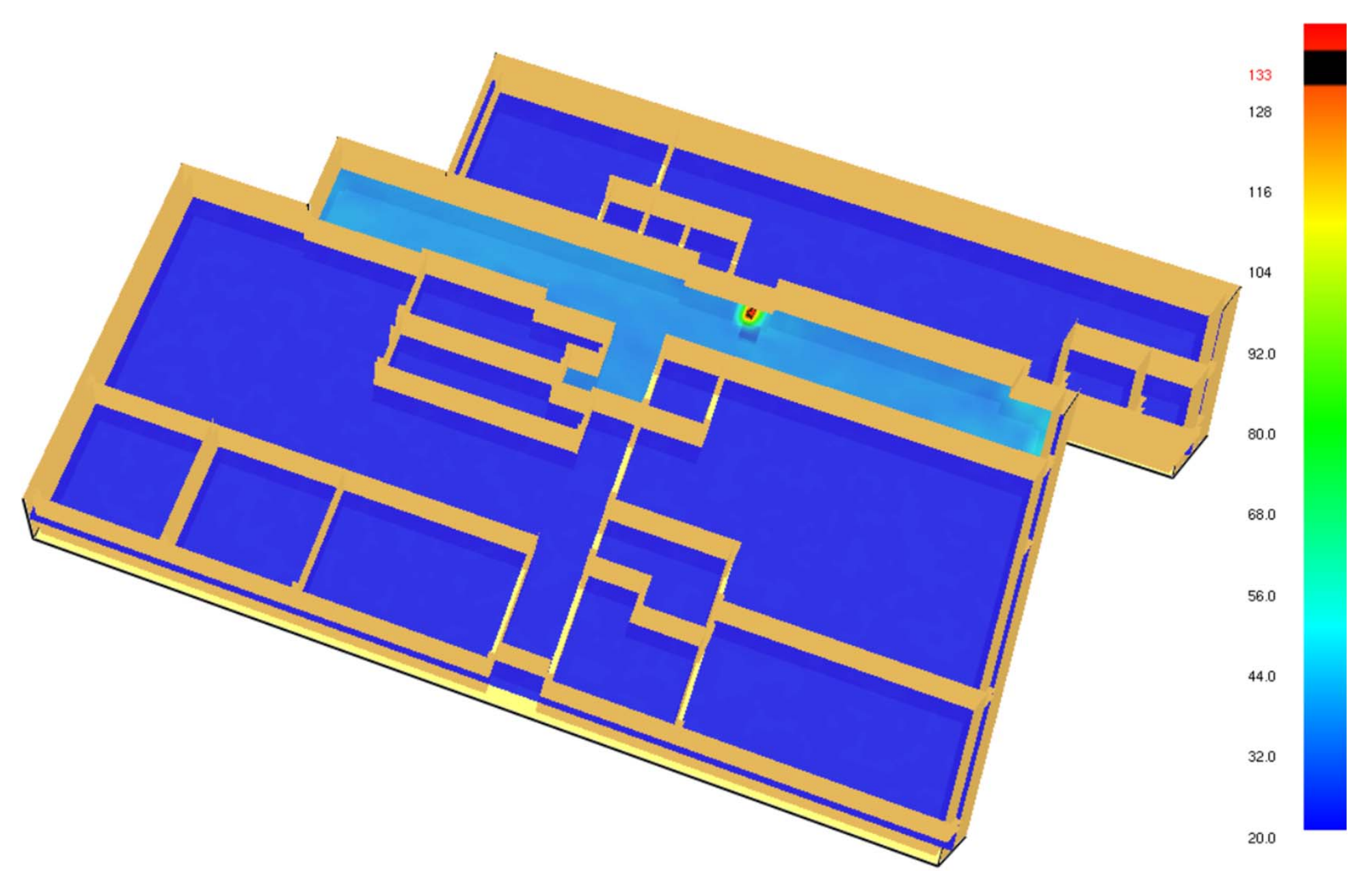

180 Seconds 


\section{Temperature- Second Floor}

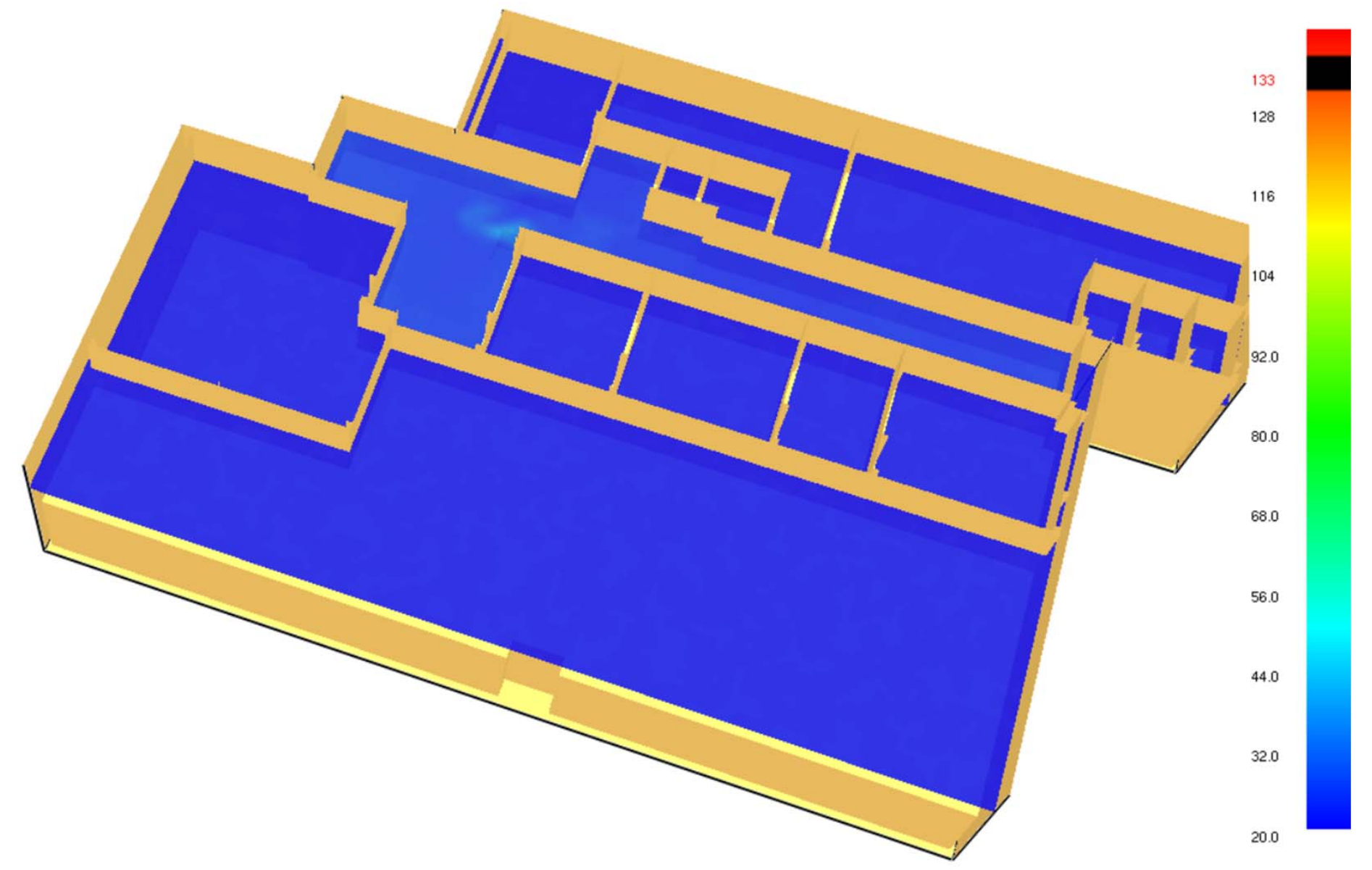

180 Seconds 


\section{Toxicity- First Floor}

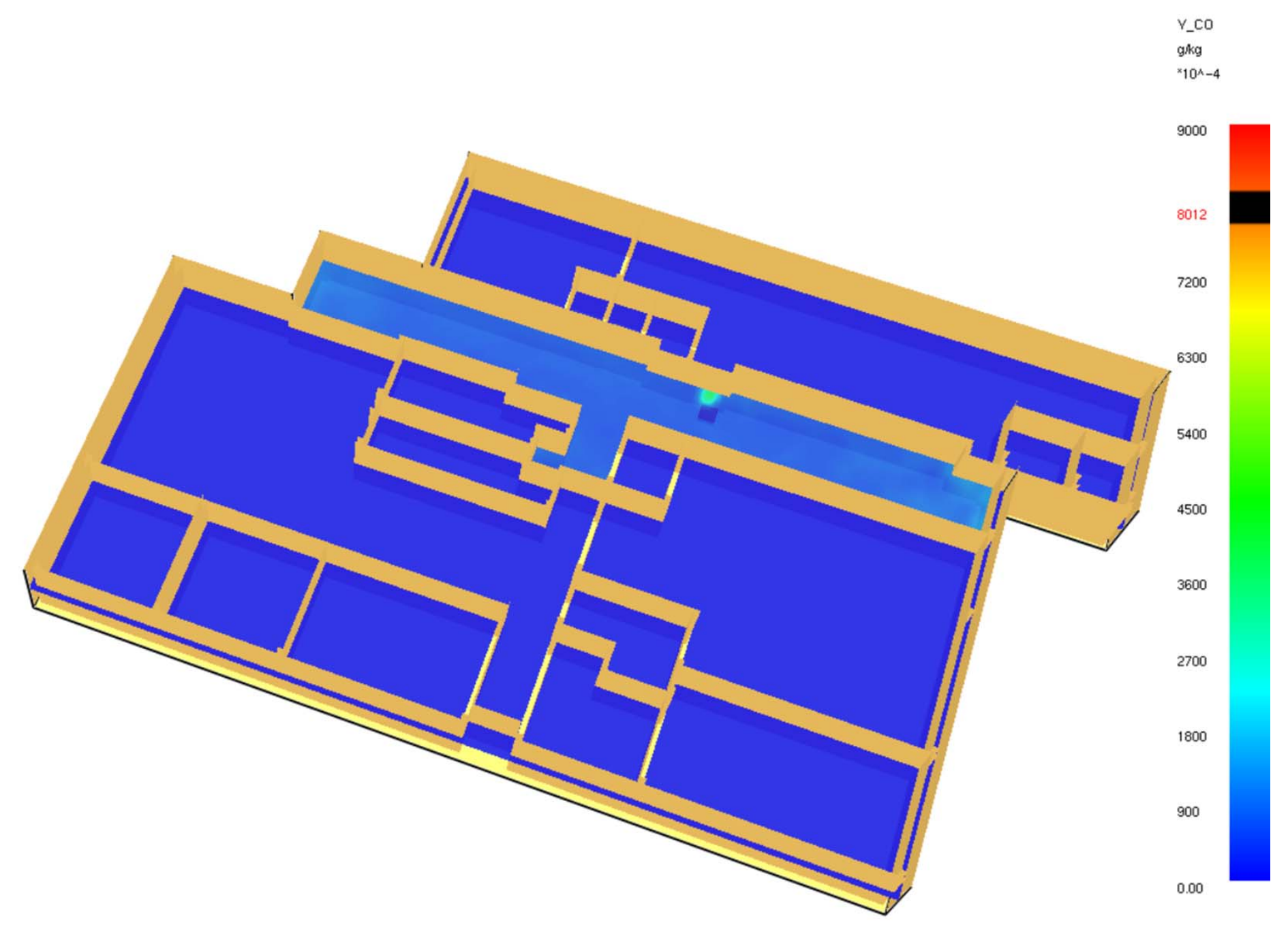

180 Seconds 


\section{Toxicity- Second Floor}

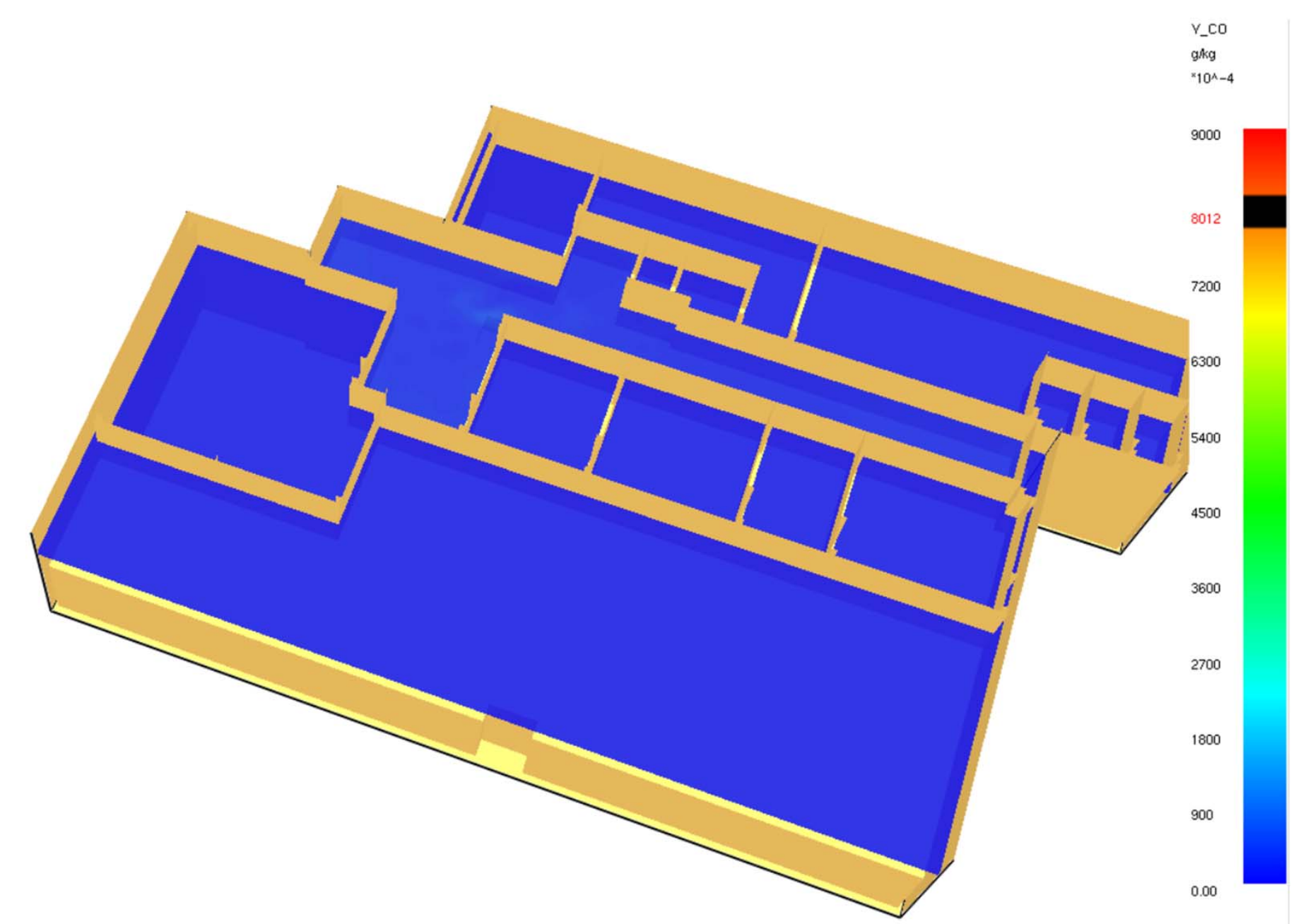

180 Seconds 


\section{ASET vs RSET Summary}

\begin{tabular}{|c|c|c|c|c|}
\hline \multirow{2}{*}{ Floor Level } & RSET Time & Visibility & Temperature & Toxicity \\
\hline Level 1 & 135 Seconds & Fail at 30 seconds & Pass & Pass \\
\hline Level 2 & 161 Seconds & Pass & Pass & Pass \\
\hline
\end{tabular}




\section{Design Fire 2- Project Integration Room}

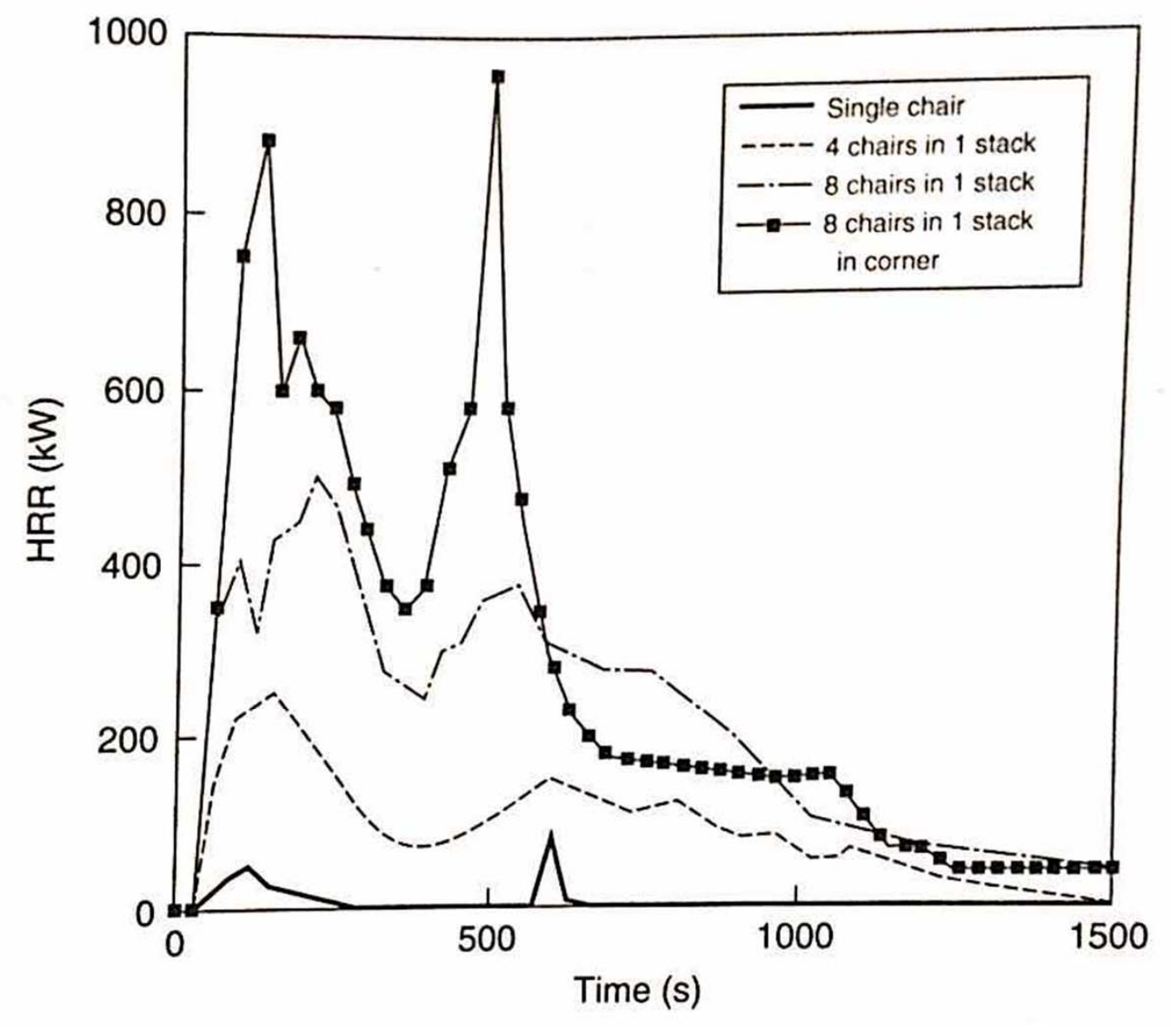

HRR of metal-frame, upholstered stacking chairs

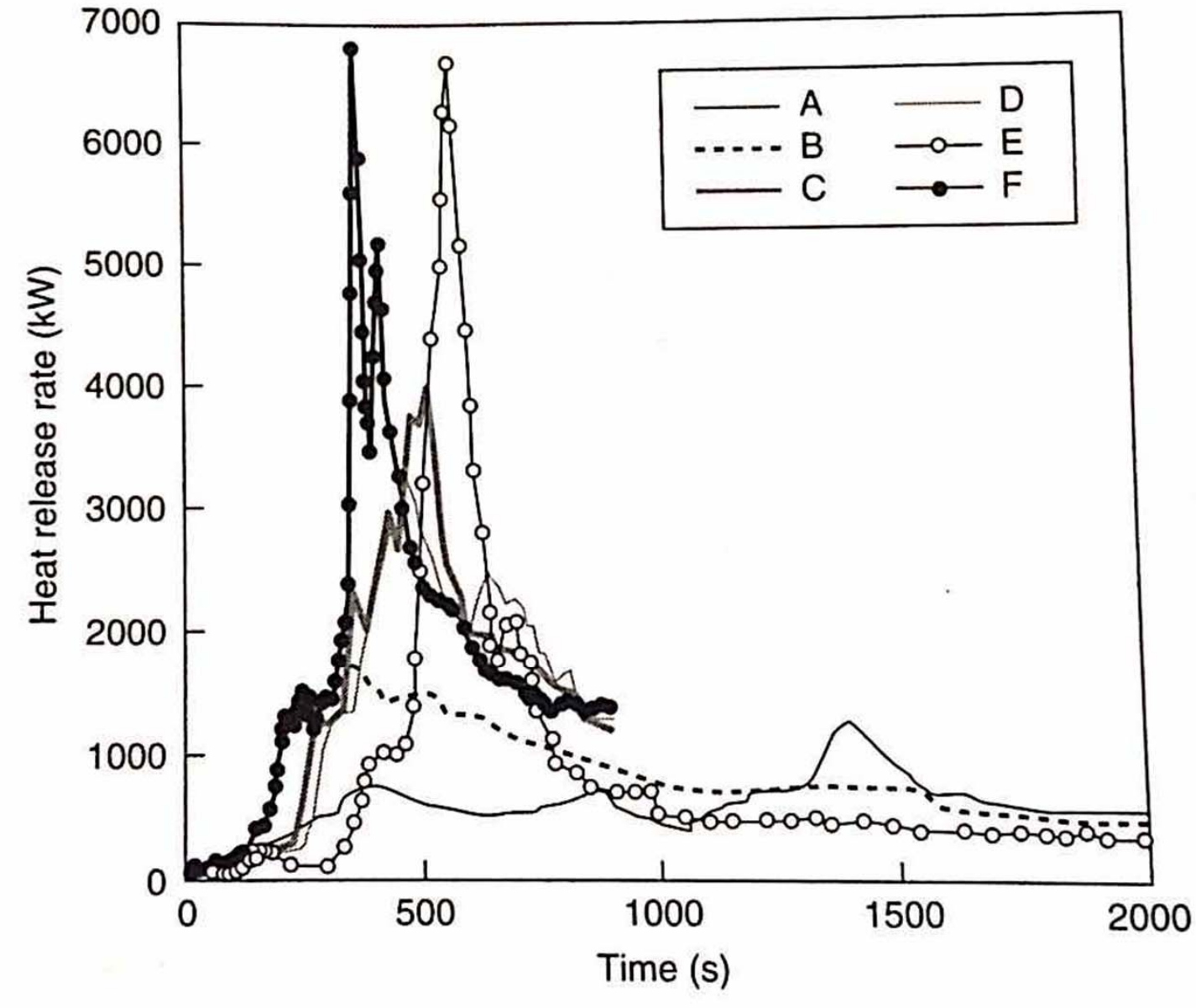

HRR of office workstations 


\section{Design Fire 2- HRR from FDS}

HRR

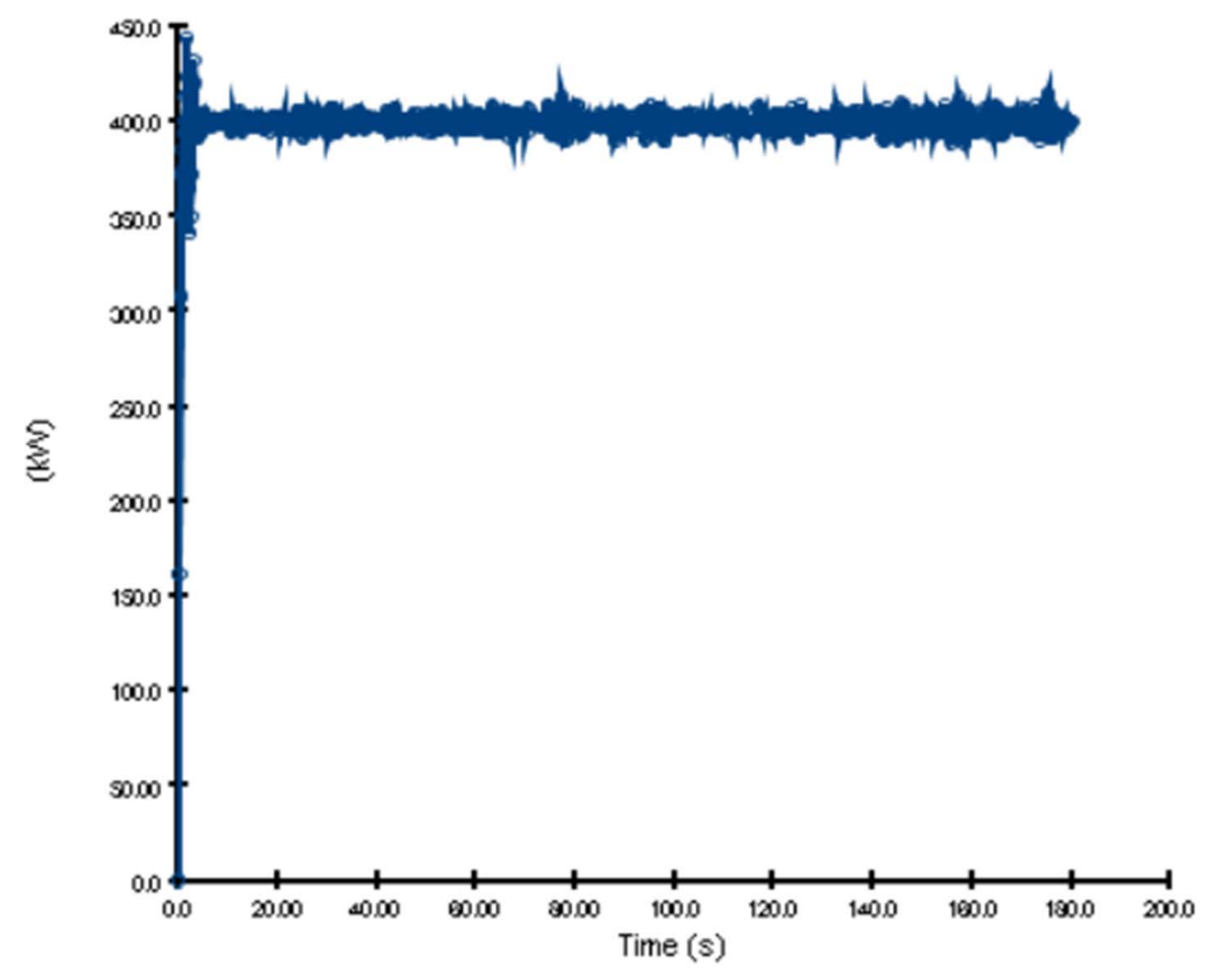

CAL POLY 


\section{Visibility- First Floor}
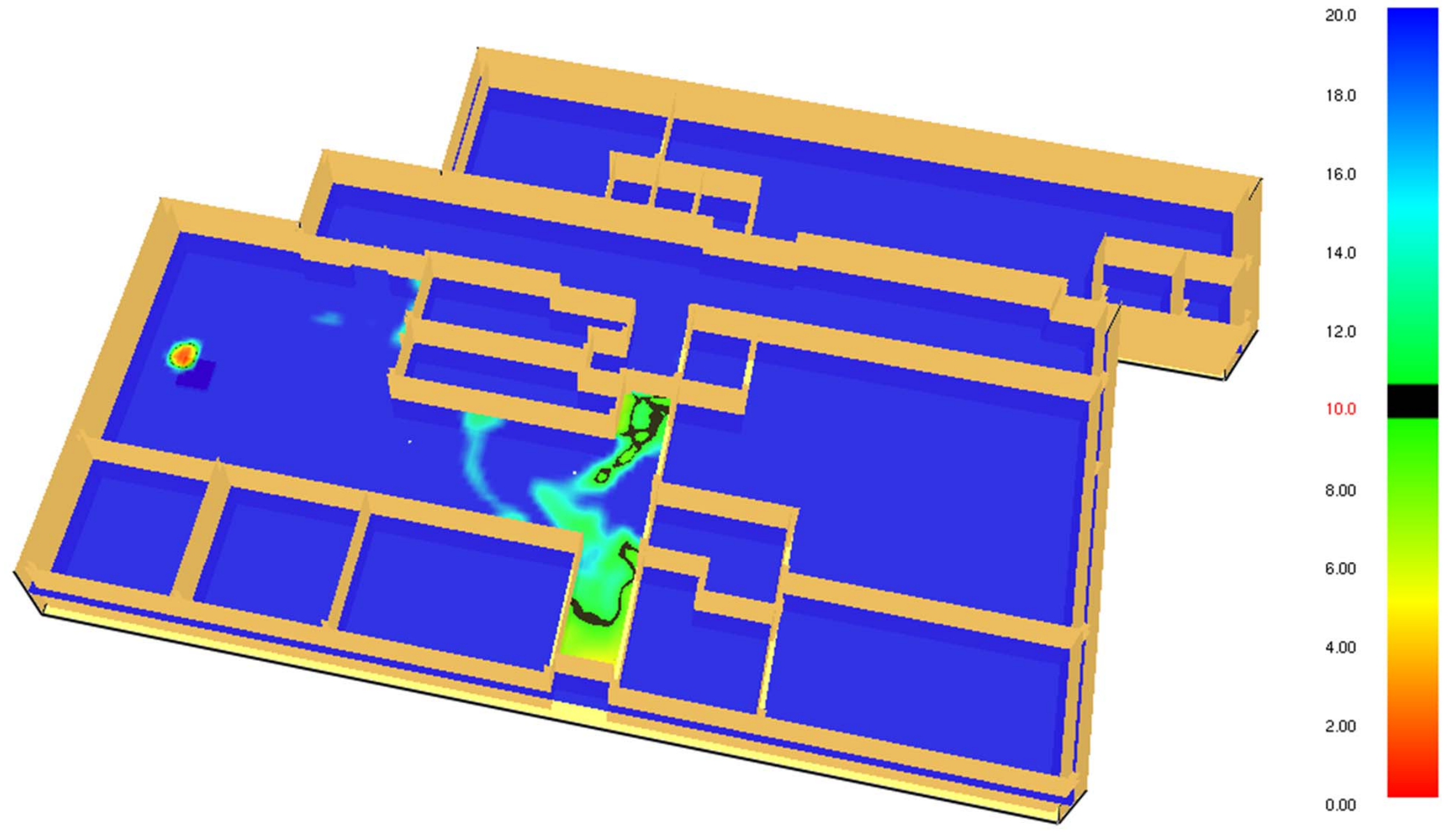

120 Seconds 


\section{Visibility- Second Floor}

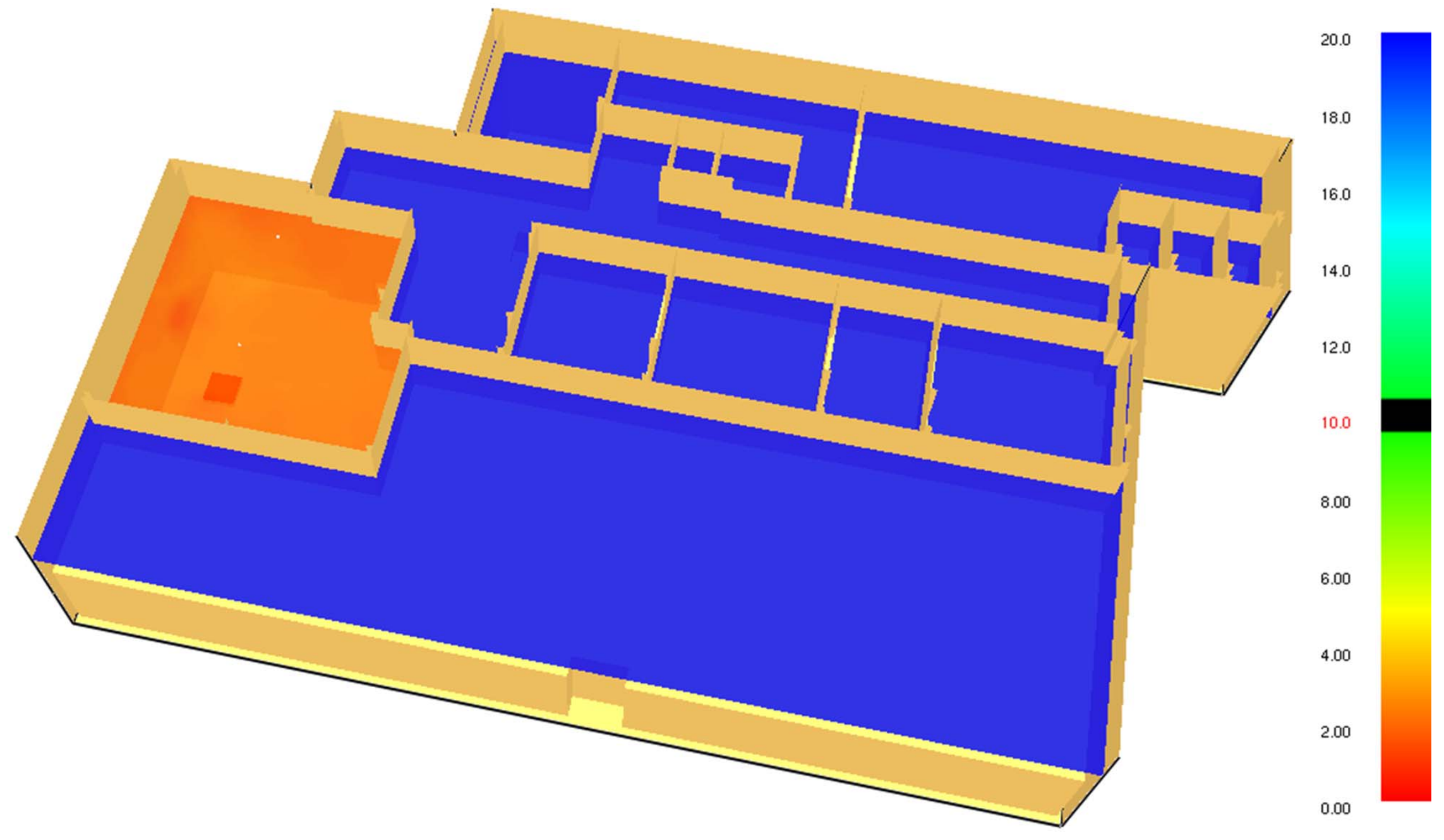

180 Seconds 


\section{Temperature- First Floor}
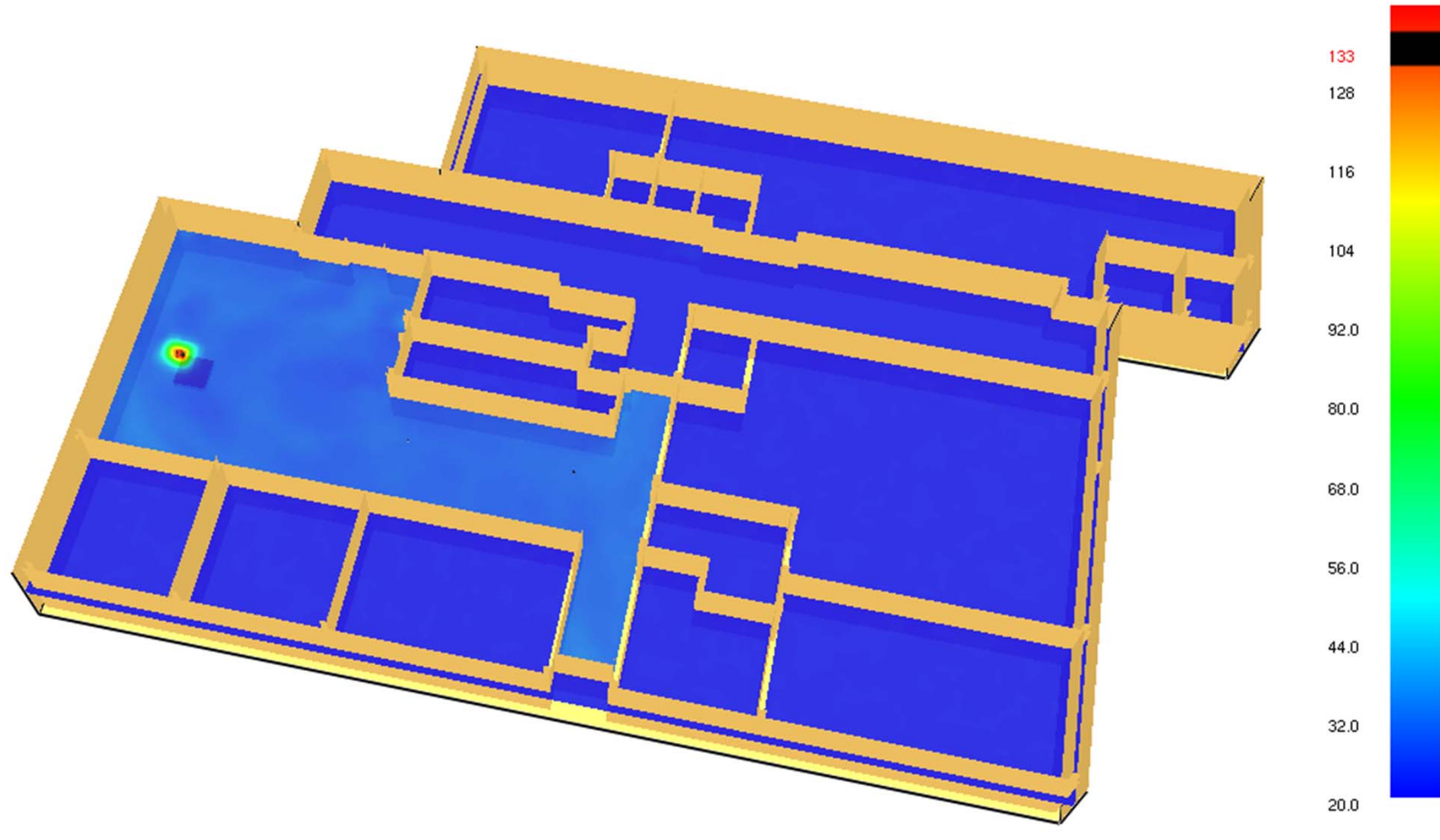

180 Seconds 


\section{Temperature- Second Floor}

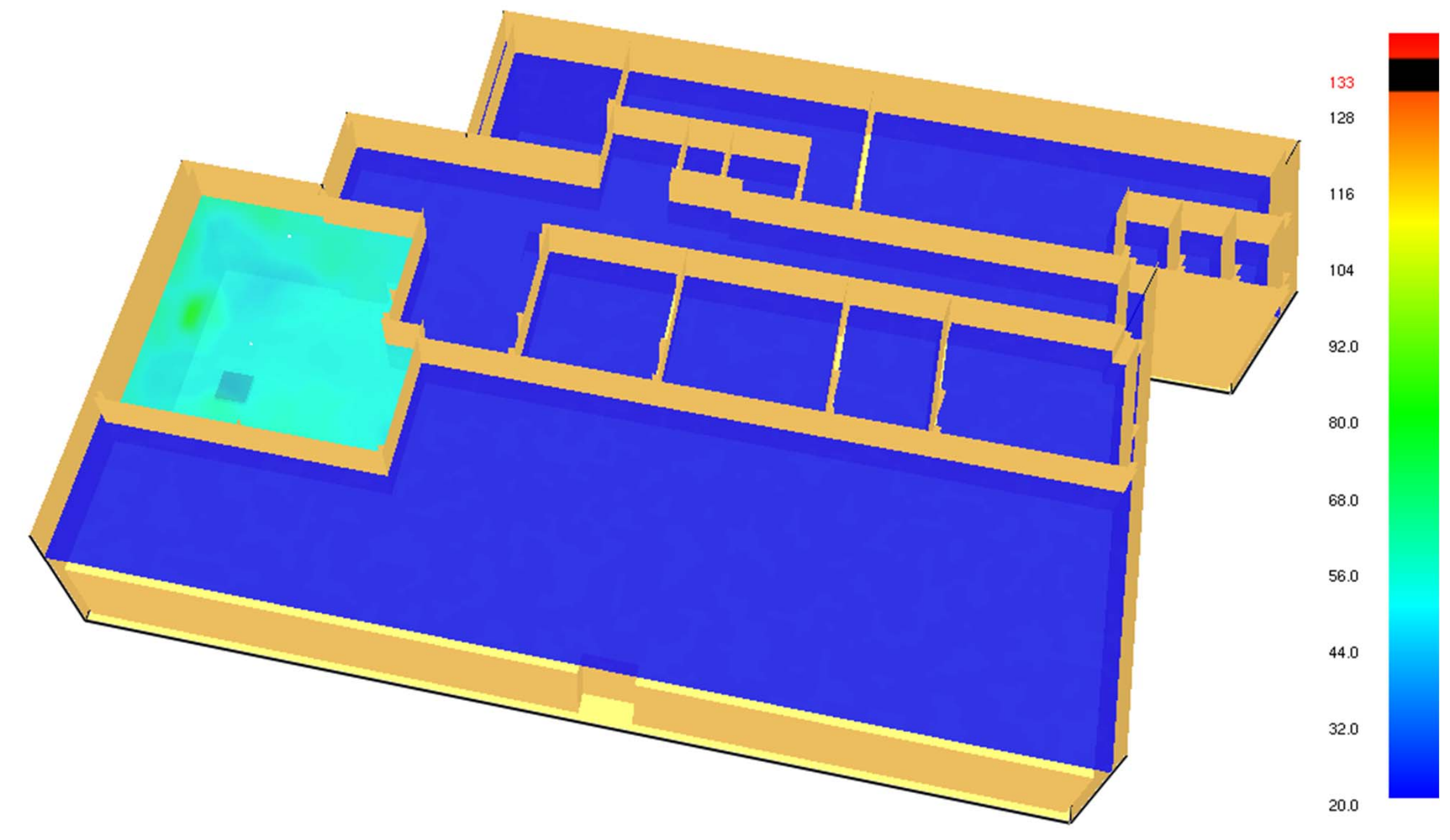

180 Seconds 
Toxicity- First Floor

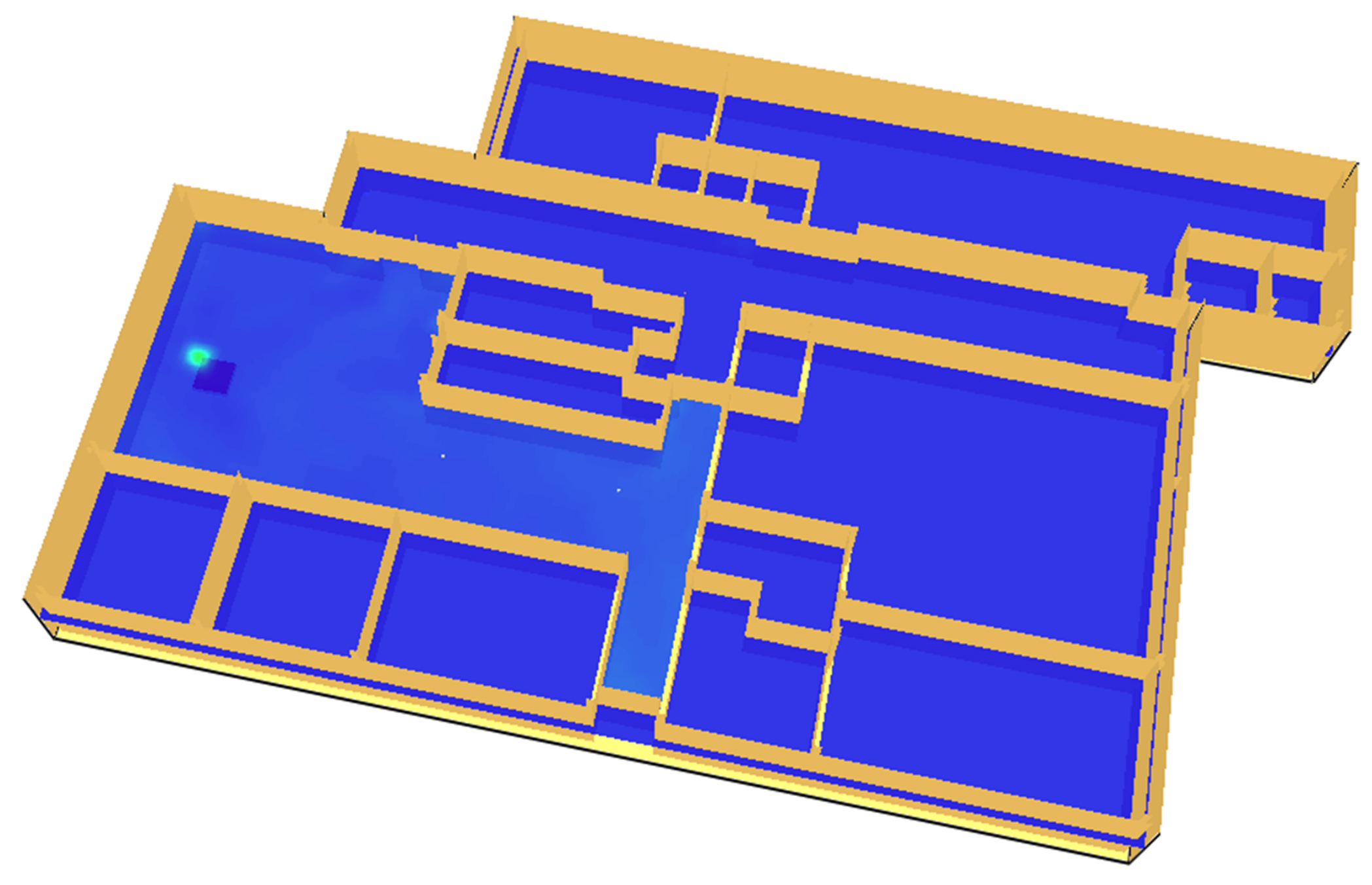

9000
8012
7200
6300
5400
4500
3600
2700
1800
900
0.00

180 Seconds

Cal Poly 


\section{Toxicity- Second Floor}

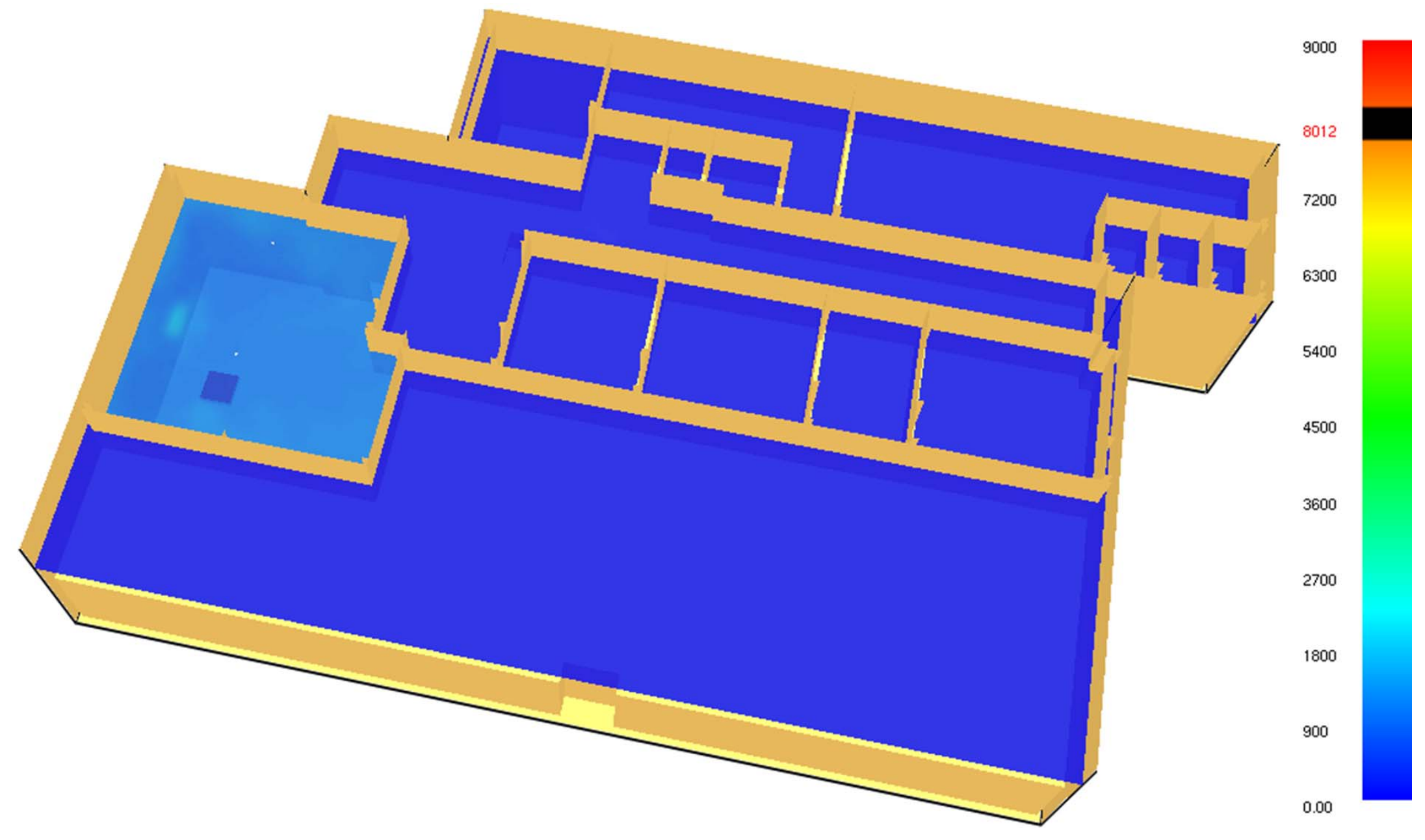

180 Seconds 


\section{ASET vs RSET Summary}

\begin{tabular}{|c|c|c|c|c|}
\hline \multirow{2}{*}{ Floor Level } & RSET Time & Visibility & Temperature & Toxicity \\
\hline Level 1 & 135 Seconds & Fail at 120 seconds & Pass & Pass \\
\hline & & & & Pass \\
\hline
\end{tabular}

- Conservative design fire and visibility parameters were chosen

- Possible solutions need to be evaluated

- Smoke control system is not required for atriums that connect only two stories (CBC 404.5 Exception). 


\section{Possible Solutions}

- Add 1 Hour Fire Barriers

- Reduce visibility tenability requirements to a minimum of 4 meters

- Design and implement a smoke control system

- Use growth and decay fire instead of steady state

- Justify use of lower soot yield 


\section{Growth and Decay instead of Steady State}
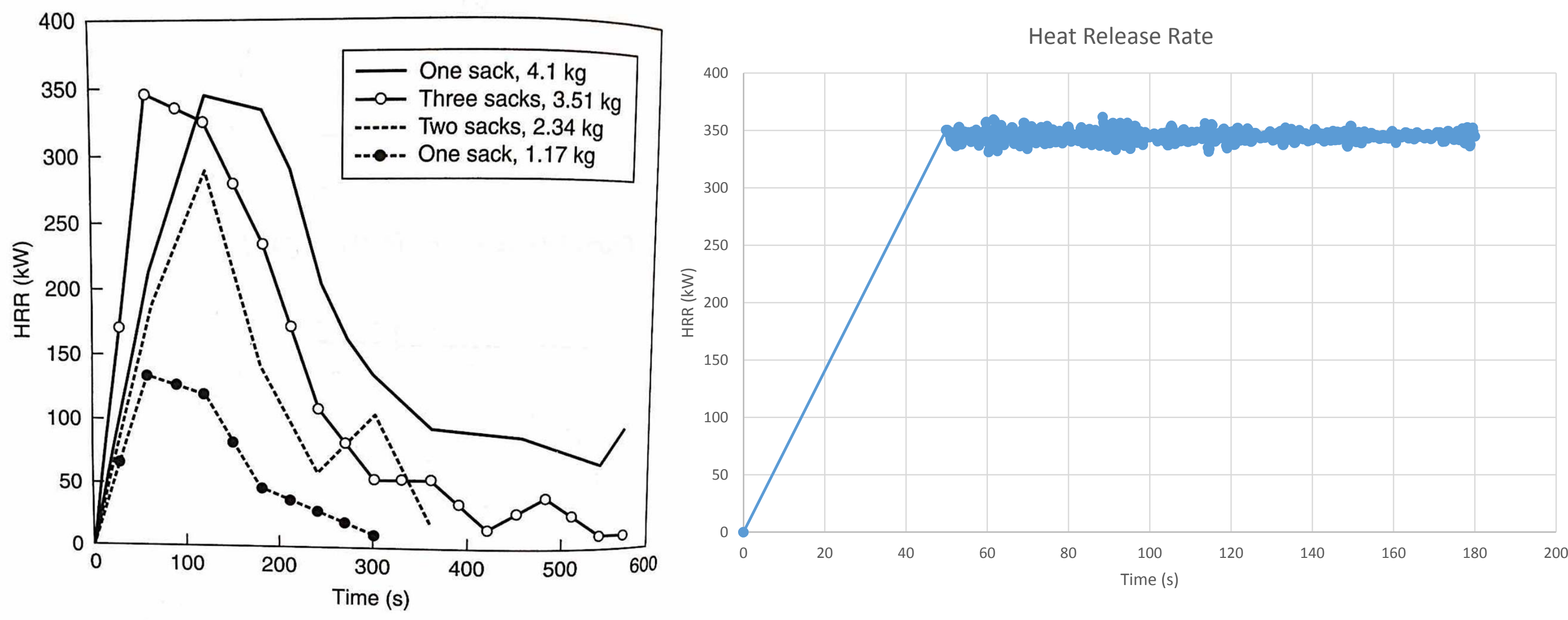

Figure 3-1.96. HRR of trash bags. 


\section{Soot Yield}

- Current Soot Yield 5\%

- Estimate contents of typical trash can

\section{Example}

Assume $50 \%$ paper and $50 \%$ plastic contents.

According to SFPE Handbook Table 3-4.16, cellulosic materials have a soot yield of 1.5\% while polyethylene has a soot yield of $6 \%$. Assuming a 50/50 ratio, the combined soot yield estimated would be $3.75 \%$. 


\section{Conclusion}

- Occupancy classification change to reflect current usage of room

- Door swing of Room 104 changed to swing in the direction of egress

- 1 Hour Fire Barrier separating atrium spaces from adjacent spaces, or approved smoke control system 


\section{Acknowledgments}

- Professor Fred Mowrer

- Professor Christopher Pascual

- Professor David Rich

- Professor Lonny Simonian

- Professor Thomas Korman

- Professor Chris Lautenberger

- Professor Francisco Joglar

- Jared Birmingham

- Northern California/Nevada Society of Fire Protection Engineers

- Fire Apparatus Manufacturers Association

- Fire Suppression System Association 